Pacific Journal of Mathematic 


\section{A CHAIN RULE FOR THE TRANSFORMATION OF INTEGRALS IN MEASURE SPACE}

\section{RoBin W. ChaneY}

This paper deals with the composition of two transformations each of which is being used to effect a transformation of an (abstract) integral by means of a change of variable. The principal result is an abstract version of the "chain rule" in a purely measure-theoretic setting. This principal result is another in the line of extensions and variations of the theorem which asserts that if $f$ and $g$ are absolutely continuous real valued functions on suitable closed intervals on the line then $g \circ f$ is absolutely continuous if and only if $\left(g^{\prime} \circ f\right) f^{\prime}$ is integrable over the domain of $f$; and if $g \circ f$ is absolutely continuous then $(g \circ f)=\left(g^{\prime} \circ f\right) f^{\prime}$. This theorem has previously been generalized to functions on $n$-space. In this paper certain results of a similar type are presented in a general measure-theoretic setting.

We shall describe the principal result of this paper somewhat more explicitly. Let $T$ be a function from a nonvoid set $\boldsymbol{S}$ onto a set $\boldsymbol{X}$. Let $\{\boldsymbol{S}, \mathfrak{M}, \boldsymbol{\mu}\}$ and $\{\boldsymbol{X}, \mathfrak{A}, \boldsymbol{\nu}\}$ be measure spaces the latter having completion $\{\boldsymbol{X}, \mathfrak{R}, \nu\}$. These entities are subjected to certain standard hypotheses such as, e.g., $T^{-1} \mathfrak{A} \subset \mathfrak{M}$ (see $\mathrm{A} 0$-A3 in 1). Within this context a nonnegative, extended real valued function ' $W$ with domain $\boldsymbol{X} \times \mathfrak{M}$ is termed a weighting function for $T$ if

$$
{ }^{\prime} W\left(., T^{-1} A\right)=0
$$

a.e. $\nu$ off $A$ for each $A$ in $\mathfrak{A}$, if ' $W(., M)=\Sigma^{\prime} W\left(., M_{i}\right)$ a.e. $\nu$ on $X$ whenever $M$ in $\mathfrak{M}$ is the union of a countable number of pairwise disjoint sets $M_{i}$ in $\mathfrak{M}$, and if each function ' $W(., M)$ is $\mathfrak{N}$-measurable; the number ' $W(x, M)$ is intended to "count" or "weigh" the number of points in $M$ mapped into $x$ by $T$. Now assume that ' $W$ is a weighting function for $T$ and let $f$ be an glb $f W$. Thus ' $W$ and $f$ bear the following relationship to each other. First, $f$ is in $L_{1}^{+}(\mu)$; moreover, if $H$ is any real valued, $\mathfrak{R}$-measurable function having domain $\boldsymbol{X}$ and $M$ is any set in $\mathfrak{M}$ then the "transformation formula"

$$
\int_{M}(H \circ T) f d \mu=\int_{X} H^{\prime} W(., M) d \nu
$$

holds as soon as one of the integrals involved exists. Now suppose also that $\Phi$ is a function from $\boldsymbol{X}$ onto $\boldsymbol{Y}$ and that $\{\boldsymbol{Y}, \mathfrak{F}, \lambda\}$ is a measure space having completion $\{\boldsymbol{Y}, \Omega, \lambda\}$; these entities are also subjected to certain standard requirements. Assume that ' $V$ is a 
weighting function for $\Phi$ and that $g$ is a $\operatorname{glb} f V$ so that ' $V$ and $g$ satisfy a transformation formula like (1). We can then discuss the possibility of obtaining a weighting function ' $U$ for $\Phi \circ T$ such that a formula like (1) holds where ' $U$ plays the role of ' $W$ and $f(g \circ T)$ plays the role of $f$. The principal theorem of this paper shows that such a ' $U$ can be obtained if and only if $f(g \circ T)$ is $\mu$-integrable; and, in case such a ' $U$ does exist it is given by an explicit formula. Indeed we have

$$
{ }^{\prime} U(y, M)=\lim _{n} \Sigma_{k} K 2^{-n \prime} V(y, E(k, n, M))
$$

a.e. $\lambda$ on $\boldsymbol{Y}$ for every $M$ in $\mathfrak{M}$, where

$$
E(k, n, M)=\left\{x \in X: k 2^{-n} \leqq{ }^{\prime} W(x, M)<(k+1) 2^{-n}\right\}
$$

for positive integers $k$ and $n$.

The theorem just described can be applied in more special settings to obtain some similar results. These theorems are, in a sense, lineal descendants of the theorem concerning the composition of absolutely continuous functions on intervals which was mentioned at the outset (see [1]). Helsel and Levine proved in [7] that if two transformations $T$ and $\Phi$ are absolutely continuous in the Banach sense (see [9]) on suitable regions in the Euclidean plane then the composition $\Phi \circ T$ is absolutely continuous in the Banach sense if and only if the product $\left(\Phi^{\prime} \circ T\right) T^{\prime}$ is Lebesgue integrable over the domain of $T$; and, if $\Phi \circ T$ is absolutely continuous then $(\Phi \circ T)^{\prime}=\left(\Phi^{\prime} \circ T\right) T^{\prime}$. In [11] Reichelderfer has generalized these results in two directions; the transformations in [11] are defined on suitable regions in Euclidean $n$-space and the concept of absolute continuity is broadened considerably. The concepts of "essential absolute continuity" and "essential jacobian" play a prominent part in [11]. In [6] Frazier has dealt with questions concerning the absolute continuity of the composition of transformations and the corresponding "chain rule" within the abstract setting described in [10]. The present paper deals with similar matters in a general measure-theoretic setting. In 3.6 we shall discuss briefly and informally the relationships among the results of these papers.

Finally we shall list the contents of the paper. The main theorem discussed above is presented early in $\S 1$. The rest of $\S 1$ is devoted to a byway; we assume that ' $U$ is a weighting function for $\Phi \circ T$ and attempt to find weighting functions ' $W$ for $T$ and ' $V$ for $\Phi$ such that ' $U,{ }^{\prime} W$, and ' $V$ are related to one another as in (2) above. The settings in which the "transformation theories" are developed in [10] and [4] are special instances of the setting of $\S 1$; in $\S 2$ we discuss the way in which the results of 1 lead to corresponding theorems in these two settings. 
In $\S 3$ the results of $\S 1$ are applied in yet another special situation in order to obtain the principal consequence of the main theorem in $\S 1$. The setting now is that of [10]. In this setting the "weight function" is the analogue of the "weighting function." We assume that $W^{\prime}$ is a weight function for $T$ and that $V^{\prime}$ is a weight function for $\Phi$, and, furthermore, that these weight functions belong to the special class discussed in $\S 7$ of [10]; this special class includes all of the (nonnegative) admissible multiplicity functions of [9] and [11] which are the forerunners and analogues of weight functions in these earlier works. Now let $f$ be a g.l.b. $f$. $W$ and $g$ be g.l.b. $f . V$. Thus $W^{\prime}$ and $f$ satisfy a formula like (1) and $V^{\prime}$ and $g$ satisfy a formula like (1). The theorem in 3.5 below asserts that there is a weight function $U^{\prime}$ for $\Phi \circ T$ of the same special type such that $f(g \circ T)$ is a g.l.b. $f . U$ if and only if $f(g \circ T)$ is integrable; again, a specific formula is given for $U^{\prime}$. It is this theorem which has the main connection with past results.

We denote the set of integers by $Z$ and the set of positive integers by $Z^{+}$. As is customary, we put $\infty \cdot 0=0$.

I wish to thank the referee for his many helpful observations; in particular, he suggested a stronger version of the main theorem and has graciously permitted me to use his proof of this stronger version.

\section{The general "chain rule" theorem.}

1. Much of the discussion in this section takes place in the setting established by imposing the standard hypotheses A0-A3. These hypotheses were first introduced in [4]. We list them for the reader's convenience.

A1. $\{\boldsymbol{S}, \mathfrak{M}, \boldsymbol{\mu}\}$ is a $\sigma$-finite, complete, positive measure space.

A2. $\{\boldsymbol{X}, \mathfrak{R}, \boldsymbol{\nu}\}$ is a $\sigma$-finite, complete, positive measure space.

A3. $T$ is a function (transformation) from $\boldsymbol{S}$ onto $\boldsymbol{X}$.

A0. $\quad\{$ is a $\sigma$-field of subsets $A$ of $X$ having the following properties. $\mathfrak{U} \subset \mathfrak{R}$ and $T^{-1} \mathfrak{U}=\left\{T^{-1} A: A \in \mathfrak{U}\right\} \subset \mathfrak{M}$. For each set $N$ in $\mathfrak{R}$ there is a set $A$ in $\mathfrak{A}$ such that $N \subset A$ and $\nu N=\nu A$. Also, (B) is a subfamily of $\mathfrak{M}$ having the property that $T \mathbb{B}=\{T G: G \in \mathbb{B}\} \subset \mathfrak{R}$.

In most of what follows, the last sentence of $\mathrm{A} 0$ is not required. We denote by $\mathrm{A} 0$ * the statement which is obtained from $\mathrm{A} 0$ by deleting the last sentence of $\mathrm{A} 0$. It is plain that if $\mathrm{A} 0 *$-A 3 (that is, $\mathrm{AO}^{*}, \mathrm{~A} 1, \mathrm{~A} 2$, and $\mathrm{A} 3$ ) are satisfied then so are $\mathrm{A} 0-\mathrm{A} 3$ if we put BS $=T^{-1} \mathfrak{A}$.

The concept of weighting function was introduced in 4.2 of [4]. We now replace the name "weighting function" by the name "BS-weighting function." We give two definitions below and warn the reader that what was formerly known as a weighting function is now 
to be called a \$s-weighting function.

Definition. Assume A0-A3. Let ' $W$ be a non negative, extended real valued function having domain $\boldsymbol{X} \times \mathfrak{M}$. ' $W$ is said to be a (S)-weighting function for $T$ if the following conditions are satisfied:

(i) If $G$ is in $\$ 5$ then ' $W(., G)=0$ a.e. $\nu$ on $C T G$. (By definition $C T G$ is the complement $X-T G$ of $T G$ relative to $X$.)

(ii) If a set $M$ in $\mathfrak{M}$ is the union of a countable number of pairwise disjoint sets $\mathbb{M}_{i}$ in $\mathfrak{M}$ then ${ }^{\prime} W(., M)=\Sigma^{\prime} W\left(., M_{i}\right)$ a.e. $\nu$ on $\boldsymbol{X}$.

(iii) For each $M$ in $\mathfrak{M}$ the function ' $W(., M)$ is $\mathfrak{R}$-measurable (on $\boldsymbol{X}$ ).

Definition. Assume A0*-A3. Let ' $W$ be a nonnegative, extended real valued function having domain $\boldsymbol{X} \times \mathfrak{M}$. ' $W$ is said to be a weighting function for $T$ if it satisfies (ii) and (iii) above and if for every $A$ in $\mathfrak{A}$ it is true that ${ }^{\prime} W\left(., T^{-1} A\right)=0$ a.e. $\nu$ on $C A$.

Definition 3. Assume A0-A3 and let ' $W$ be a \$3-weighting function for $T$. Define a nonnegative, extended real valued function $W$ having domain $\mathfrak{M}$ by putting $W M=\int_{\boldsymbol{X}} W(., M) d \nu$ for every $M$ in M. The extended real number $W M$ is the weight attached to $M$ by ' $W$.

Definitions 4. Assume A0-A3. Define $L_{1}^{+}(\mu)$ to be the set of all nonnegative functions in the space $L_{1}(\mu)=L_{1}(\{\boldsymbol{S}, \mathfrak{M}\{\mu\})$ of all extended real valued, $\mu$-integrable functions on $S$. Define $J(T, \mu, \nu)=$ $J(T)$ to be the set of all functions $f$ in $L_{1}^{+}(\mu)$ which have this property: If $N$ is a $\nu$-null set in $\mathfrak{R}$ then $f=0$ a.e. $\mu$ on $T^{-1} N$. Finally define $J^{\prime}(T, \mu, \mathbb{S})=J^{\prime}(T$, (S) to be the set of all (S)-weighting functions ' $W$ for $T$ for which there exists a function $f$ in $J(T)$ such that

(i) $\int_{M} f d \mu=W M=\int_{X}{ }_{X} W(., M) d \nu$ for every $M$ in $\mathfrak{M}$;

(ii) the transformation formula holds for ' $W$ and $f$; that is, let $M$ be any set in $\mathfrak{M}$ and let $H$ be any real valued, $\mathfrak{R}$-measurable function having domain $X$ : then $(H \circ T) f$ is $\mathfrak{M}$-measurable (cf. 2.13 of [10]) and $H^{\prime} W(., M)$ is $\Re$-measurable; if $(H \circ T) f$ is $\mu$-integrable over $M$ or if $H^{\prime} W(., M)$ is in $L_{1}(\nu)$ then $(H \circ T) f$ is $\mu$-integrable over $M$, $H^{\prime} W(., M)$ is in $L_{1}(\nu)$, and

$$
\int_{X} H^{\prime} W(., M) d \nu=\int_{M}(H \circ T) f d \mu .
$$

Given ' $W$ in $J^{\prime}(T$, (S), any function $f$ in $J(T)$ for which (i) and (ii) hold is termed a greatest lower bound function for the weights $W$ induced by ' $W$-briefly, a g.1.b. f. $W$. 
Note 5. A0*-A3. If we put $\mathbb{B}=T^{-1} \mathfrak{A}$, then $\mathrm{A} 0$ is satisfied and any weighting function for $T$ is a $\$$-weighting function for $T$. In case $\mathbb{B}=T^{-1 \mathfrak{Q}}$ we denote $J^{\prime}\left(T\right.$, (S) by $J^{\prime}(T)$.

Lemma 6. Assume A0-A3 and let ' $W_{1}$ be in $J^{\prime}\left(T\right.$, (8) and ' $W_{2}$ be in $J^{\prime}\left(T\right.$, (S). Assume that ' $W_{1}(., M) \leqq{ }^{\prime} W_{2}(., M)$ a.e. $\nu$ on $\boldsymbol{X}$ for every $M$ in $\mathfrak{M}$. If $f_{1}$ is a g.l.b. $f . W_{1}$ and if $f_{2}$ is a g.l.b. $f . W_{2}$ then $f_{1} \leqq f_{2}$ a.e. $\mu$ on $S$.

Lemma 7. Assume A0-A3 and that ' $W_{1}$ and ' $W_{2}$ are in $J^{\prime}(T$, \&). Let $f_{1}$ be a g.l.b. $f . W_{1}$ and $f_{2}$ be a g.l.b. $f . W_{2}$. If $f_{1} \leqq f_{2}$ a.e. $\mu$ on $\boldsymbol{S}$ then ' $W_{1}(., M) \leqq{ }^{\prime} W_{2}(., M)$ a.e. $\nu$ on $\boldsymbol{X}$ for every $M$ in $\mathfrak{M}$.

Proof. Let $M$ be in $\mathfrak{M}$. Let $N$ be any set in $\mathfrak{R}$ and let $h$ be the characteristic function of $N$ as a subset of $\boldsymbol{X}$. Then, according to the transformation formula, we have

$$
\begin{aligned}
& \int_{N}{ }^{\prime} W_{1}(., M) d \nu=\int_{M}(h \circ T) f_{1} d \mu \\
& \quad \leqq \int_{M}(h \circ T) f_{2} d \mu=\int_{N}{ }^{\prime} W_{2}(., M) d \nu .
\end{aligned}
$$

The desired conclusion follows at once.

REMARKs 8. Assume A0-A3. According to 4.6 of [4], it is true that for every $f$ in $J(T)$ there exists ' $W$ in $J^{\prime}(T$, (S) such that $f$ is a g.l.b. $f W$.

Lemma 9. Assume A0*-A3. Suppose that ' $W$ is a weighting function for $T$. Then ' $W(., M)=0$ a.e. ע on $C T M$ for every $M$ in $\mathfrak{M}$ such that $T M$ is in $\mathfrak{R}$.

Proof. Given $M$ in $\mathfrak{M}$ for which $T M$ is in $\mathfrak{R}$, select $A$ in $\mathfrak{A}$ so that $T M \subset A$ and $\nu(A \cap C T M)=0$. Then ${ }^{\prime} W\left(., T^{-1} A\right)=0$ a.e. $\nu$ on CTM. But $M \subset T^{-1} A$ and so ${ }^{\prime} W(., M) \leqq{ }^{\prime} W\left(., T^{-1} A\right)$ a.e. $\nu$ on $\boldsymbol{X}$. Hence ' $W(., M)=0$ a.e. $\nu$ on $C T M$.

Theorem 10. Assume A0-A3. Then $J^{\prime}(T)=J^{\prime}(T$, (8)).

Proof. First suppose that ' $W$ is in $J^{\prime}(T)$. It follows from .9 that ' $W(., G)=0$ a.e. $\nu$ on $C T G$ for every $G$ in $\$$ (s). Therefore ' $W$ is a (S)-weighting function for $T$ and ' $W$ is in $J^{\prime}(T$, (S).

Now assume that ' $W$ is in $J^{\prime}(T$, (B). If we can prove that ' $W$ is a weighting function for $T$ then it will follow that ' $W$ is in $J^{\prime}(T)$. Thus, let $A$ be a set in $\mathfrak{A}$. If $f$ is a g.l.b. $f . W$. and if $H$ is the characteristic function of $C A$ then 


$$
\begin{aligned}
\int_{C A} W\left(., T^{-1} A\right) d \nu & =\int_{X} H^{\prime} W\left(., T^{-1} A\right) d \nu=\int_{T^{-1} A}(H \circ T) f d \mu \\
& =\int_{T^{-1} A_{A} \cap T^{-1} C A} f d \mu=0 .
\end{aligned}
$$

As a result, ' $W\left(., T^{-1} A\right)=0$ a.e. $\nu$ on $C A$.

REMARK. Thus the set $J^{\prime}(T$, (S) actually does not depend on (S) and, in fact, is identical to $J^{\prime}(T)$. In the sequel we shall always consider $J^{\prime}(T)$ rather than $J^{\prime}(T$, (S).

Lemma 11. Assume A0*-A3. Assume that ' $W$ is a weighting function for $T$ and suppose that there is a function $f$ in $L_{1}^{+}(\mu)$ for which

$$
\int_{M} f d \mu=\int_{X}{ }^{\prime} W(., M) d \nu
$$

for every $M$ in $\mathfrak{M}$. Then $f$ is in $J(T),{ }^{\prime} W$ is in $J^{\prime}(T)$, and $f$ is a g.l.b. $f . W$.

Proof. First we prove that $f$ is in $J(T)$. Let $N$ be a $\nu$-null set in $\mathfrak{R}$. There is a $\nu$-null set $A$ in $\mathfrak{A}$ such that $N \subset A$. Then

$$
\int_{T^{-1} A} f d \mu=\int_{A}^{\prime} W\left(., T^{-1} A\right) d \nu+\int_{C A}{ }^{\prime} W\left(., T^{-1} A\right) d \nu=0+0 .
$$

To complete the proof, we must show that ' $W$ and $f$ satisfy the transformation formula (see 4). Let $H$ be the characteristic function of a set $A$ in $\mathfrak{U}$ and let $M$ be in $\mathfrak{M}$. Then

$$
\begin{gathered}
\int_{X} H^{\prime} W(., M) d \nu=\int_{X} H^{\prime} W\left(., M \cap T^{-1} A\right) d \nu+\int_{X} H^{\prime} W\left(., M \cap T^{-1} C A\right) d \nu \\
\quad=\int_{A} H^{\prime} W\left(., M \cap T^{-1} A\right) d \nu+\int_{C A} H^{\prime} W\left(., M \cap T^{-1} C A\right) d \nu \\
\quad=\int_{A} W\left(., M \cap T^{-1} A\right) d \nu=\int_{X} W\left(., M \cap T^{-1} A\right) d \nu=\int_{M \cap T_{1}^{-1} A} f d \mu .
\end{gathered}
$$

Next let $H$ be the characteristic function of a set $N$ in $\Re$ and let $M$ be in $\mathfrak{M}$. There is a set $A$ in $\mathfrak{U}$ such that $N \subset A$ and $\nu(A \cap C N)=0$. Denote by $H_{1}$ the characteristic function of $A$. Since $f$ is in $J(T)$ we have

$$
\int_{M}(H \circ T) f d \mu=\int_{M}\left(H_{1} \circ T\right) f d \mu=\int_{X} H_{1}^{\prime} W(., M) d \nu=\int_{X} H^{\prime} W(., M) d \nu .
$$

Now that the transformation formula is proved for characteristic functions of sets in $\mathfrak{R}$, we can complete the proof that the transformation formula holds by using familiar theorems and arguments from measure theory. 
LEMMA 12. Assume A0*-A3. Assume that ' $W$ is a weighting function for $T$ and suppose that $\left\{E_{m}\right\}$ is a sequence of sets in $\mathfrak{M}$ for which $E_{m} \subset E_{m+1}$ for all $m$ in $Z^{+}$. Then

$$
' W\left(., \cup E_{m}\right)=\lim _{m} ' W\left(., E_{m}\right)
$$

a.e. $\nu$ on $\boldsymbol{X}$.

The proof is virtually the same as the familiar proof that, under these conditions on $\left\{E_{m}\right\}, \mu\left(\bigcup E_{m}\right)=\lim _{m} \mu E_{m}$.

LEMma 13. Assume A0*-A3.

(a) Assume that $\left\{{ }^{\prime} W_{n}\right\}$ is a sequence of weighting functions for $T$ such that ' $W_{n}(., M) \leqq{ }^{\prime} W_{n+1}(., M)$ a.e. $\nu$ on $\boldsymbol{X}$ fo every $M$ in Me. Define a function ' $W$ on $\boldsymbol{X} \times \mathfrak{M i}$ by putting

$$
{ }^{\prime} W(x, M)=\lim _{n}{ }^{\prime} W_{n}(x, M)
$$

whenever this limit exists and setting ' $W(x, M)=0$ otherwise. Then ' $W$ is a weighting function for $T$.

(b) Assume, in addition, that each ' $W_{n}$ is in $J^{\prime}(T)$ and for $n$ in $Z^{+}$let $f_{n}$ be a g.l.b. $f . W_{n}$. Then $f_{n} \leqq f_{n+1}$ a.e. $\mu$ on $\boldsymbol{S}$ for each $n$. Define a function $f$ having domain $\boldsymbol{S}$ by putting $f(s)=\lim _{n} f_{n}(s)$ if $\lim _{n} f_{n}(s)$ exists and setting $f(s)=0$ otherwise. Then' $W$ is in $J^{\prime}(T)$ if and only if 'W(., $S)$ is in $L_{1}^{+}(\nu)$, or, equivalently, if and only if $f$ is in $L_{1}^{+}(\mu)$. And if ' $W$ is in $J^{\prime}(T)$ then $f$ is a g.1.b. $f . W$.

The proof is routine and we omit it. For the proof of (b) one can use .6 and .11.

Remarks 14. The standard hypotheses $\mathrm{A} 0 *$-A3 set forth above are not sufficient for the present study because we are now concerned with the composition $\Phi \circ T$ of two transformations $T$ and $\Phi$. Hence we introduce two additional lists of statements denoted by B0*-B3 and $\mathrm{C} 0{ }^{*}-\mathrm{C} 3$. The statements $\mathrm{B} 0{ }^{*}-\mathrm{B} 3$ are obtained from the statements A0*-A3 by replacing $\{\boldsymbol{S}, \mathfrak{M}, \mu\}$ by $\{\boldsymbol{X}, \mathfrak{R}, \boldsymbol{\nu}\},\{\boldsymbol{X}, \mathfrak{R}, \boldsymbol{\nu}\}$ by $\{\boldsymbol{Y}, \Re, \lambda\}, T$ by $\Phi$, and $\mathfrak{U}$ by $\mathfrak{F}$ throughout the statements $\mathrm{A} 0 *-\mathrm{A} 3$. The statements $\mathrm{C} 0 *$-C3 are obtained from the statements $\mathrm{A} 0 *$-A3 by replacing $\{\boldsymbol{X}, \mathfrak{N}, \boldsymbol{\nu}\}$ by $\{\boldsymbol{Y}, \Re, \lambda\}, T$ by $\Phi \circ T$, and $\mathfrak{U}$ by $\mathfrak{F}$ throughout the statements $\mathrm{A} 0 *-\mathrm{A} 3$.

It is easy to check that all of the statements $\mathrm{A} 0 *-\mathrm{A} 3, \mathrm{~B} 0^{*}-\mathrm{B} 3$, and $\mathrm{C} 0 *-\mathrm{C} 3$ are satisfied if one assumes merely $\mathrm{A} 0^{*}-\mathrm{A} 3, \mathrm{~B} 0^{*}, \mathrm{~B} 2, \mathrm{~B} 3$, and the inclusion $(\Phi \circ T)^{-1} \mathfrak{F} \subset \mathfrak{M}$.

Theorem 15. Assume $\mathrm{A} 0 *-\mathrm{A} 3, \mathrm{~B} 0 *-\mathrm{B} 3$, and $\mathrm{C} 0$ *-C3. Assume that 
' $W$ is in $J^{\prime}(T)$ and that ' $V$ is in $J^{\prime}(\Phi)$. Then, for each $M$ in $\mathfrak{M}$ and for every pair $k$ and $n$ in $Z^{+}$define

$$
E(k, n, M)=\left\{x \in \boldsymbol{X}: k 2^{-n} \leqq ' W(x, M)<(k+1) 2^{-n}\right\} .
$$

For each $M$ in $\mathfrak{M}$ there exists a $\lambda$-null set $K(M)$ in $\Re$ such that the sequence $\left\{\Sigma_{k} k 2^{-n \prime} V(y, E(k, n, M)\}_{n}\right.$ is nondecreasing for every $y$ in $\boldsymbol{Y}-K(M)$. Define a nonnegative, extended real valued function 'U having domain $\boldsymbol{Y} \times \mathfrak{M}$ by putting

$$
{ }^{\prime} U(y, M)=\lim _{n} \Sigma_{k} k 2^{-n \prime} V(y, E(k, n, M))
$$

if $y$ is in $\boldsymbol{Y}-K(M)$ and ' $U(y, M)=0$ if $y$ is in $K(M)$. Then ' $U$ is a weighting function for $\Phi \circ T$.

Let $f$ be a g.l.b. $f . W$. and $g$ be an g.l.b. $f . V$. Then' $U(., S)$ is in $L_{1}^{+}(\lambda)$ if and only if $g^{\prime} W(., S)$ is in $L_{1}^{+}(\nu)$, or equivalently, if and only if $f(g \circ T)$ is in $L_{1}^{+}(\mu)$. If ' $U(., S)$ is in $L_{1}^{+}(\lambda)$ then ' $U$ is in $J^{\prime}(\Phi \circ T)$ and $f(g \circ T)$ is a g.l.b. $f . U$.

Proof. For every $m$ in $Z^{+}$define $E_{m}=\left\{x \in \boldsymbol{X}:{ }^{\prime} W(x, \boldsymbol{S})<2^{m}\right\}$. The sets $E(k, n, M)$ and $E(k, n, M) \cap E_{m}$ are all in $\mathfrak{N}$ so that the expressions ' $V(y, E(k, n, M))$ and ' $V\left(y, E(k, n, M) \cap E_{m}\right)$ are always defined. For each pair $m$ and $n$ in $Z^{+}$define a function $g_{m n}$ having domain $\boldsymbol{Y} \times \mathfrak{M}$ by putting

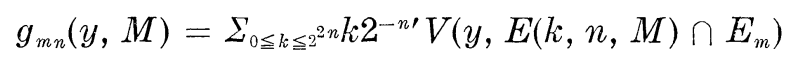

for every $(y, M)$ in $\boldsymbol{Y} \times \mathfrak{M}$. Each $g_{m n}(., M)$ is in $L_{1}^{+}(\lambda)$ and we have

$$
\int_{F} g_{m n}(., M) d \lambda=\Sigma_{0 \leqq k \leqq 22 n} \int_{E(k, n, M) \cap E_{m} \cap \Phi^{-1} F} k 2^{-n} g d \nu
$$

for every $F$ in $\mathfrak{F}$. It is clear that $E(k, n, M)$ is the union of the disjoint sets $E(2 k, n+1, M)$ and $E(2 k+1, n+1, M)$; hence

${ }^{\prime} V(y, E(k, n, M))={ }^{\prime} V(y, E(2 k, n+1, M))+{ }^{\prime} V(y, E(2 k+1, n+1, M))$

a.e. $\lambda$ on $Y$ and

$$
\begin{aligned}
& { }^{\prime} V\left(y, E(k, n, M) \cap E_{m}\right)={ }^{\prime} V\left(y, E(2 k, n+1, M) \cap E_{m}\right) \\
& \quad+{ }^{\prime} V\left(y, E(2 k+1, n+1, M) \cap E_{m}\right)
\end{aligned}
$$

a.e. $\lambda$ on $\boldsymbol{Y}$ for every $k, m, n$ and $M$. It follows from .12 that $\lim _{m}{ }^{\prime} V\left(y, E(k, n, M) \cap E_{m}\right)={ }^{\prime} V(y, E(k, n, M))$ a.e. $\lambda$ on $\boldsymbol{Y}$ for every $k, n$, and $M$. Hence for every $M$ in $\mathfrak{M}$ there is a $\lambda$-null set $K(M)$ in $\Re$ such that the relations 


$$
\begin{aligned}
\prime V(y, E(k, n M)) & ={ }^{\prime} V(y, E(2 k, n+1, M)) \\
& +{ }^{\prime} V(y, E(2 k+1, n+1, M)) ; \\
' V\left(y, E(k, n, M) \cap E_{m}\right) & =' V\left(y, E(2 k, n+1, M) \cap E_{m}\right) \\
& +{ }^{\prime} V\left(y, E(2 k+1, n+1, M) \cap E_{m}\right) ; \\
{ }^{\prime} V\left(y, E(k, n, M) \cap E_{m}\right) & \leqq ' V\left(y, E(k, n, M) \cap E_{m+1}\right) ; \\
\lim _{m}{ }^{\prime} V\left(y, E(k, n, M) \cap E_{m}\right) & ={ }^{\prime} V(y, E(k, n, M))
\end{aligned}
$$

all hold for every $k, n, m$ in $Z^{+}$and $y$ in $Y-K(M)$. It follows that $g_{m n}(y, M) \leqq g_{m+1, n}(y, M)$ and $g_{m n}(y, M) \leqq g_{m, n+1}(y, M)$ hold for every $m$ and $n$ in $Z^{+}$and $y$ in $\boldsymbol{Y}-K(M)$.

At this point we define functions $g_{m}$ having domains $\boldsymbol{Y} \times \mathfrak{M}$ by putting $g_{m}(y, M)=\lim _{n} g_{m n}(y, M)$ for $y$ in $\boldsymbol{Y}-K(M)$ and putting $g_{m}(y, M)=0$ for $y$ in $K(M)$. Each $g_{m}(., M)$ is $\Omega$-measurable and we have $g_{m} \leqq g_{m+1}$ for every $m$ in $Z^{+}$. Now define a function ' $U$ having domain $\boldsymbol{Y} \times \mathfrak{M}$ by putting ' $U(y, M)=\lim _{m} g_{m}(y, M)$ for every $(y, M)$ in $\boldsymbol{Y} \times \mathfrak{M}$. For $M$ in $\mathfrak{M}$ and $y$ in $\boldsymbol{Y}-K(M)$ we have

$$
\begin{aligned}
{ }^{\prime} U(y, M) & =\lim _{m} g_{m}(y, M) \\
& =\lim _{n} \lim _{m} g_{m n}(y, M) \\
& =\lim _{n} \Sigma_{0 \leqq k \leqq 22 n} k 2^{-n \prime} V(y, E(k, n, M)) \\
& =\lim _{n} \Sigma_{k} k 2^{-n \prime} V(y, E(k, n, M)) .
\end{aligned}
$$

We shall denote the characteristic function of a set $N$ in $\mathfrak{R}$ by $H(., N)$. For every pair $m$ and $n$ in $Z^{+}$define a function $h_{m n}$ having domain $\boldsymbol{X} \times \mathfrak{M}$ by setting

$$
h_{m n}(x, M)=\Sigma_{0 \leqq k \leqq 2 n} k 2^{-n} H\left(x, E(k, n, M) \cap E_{m}\right)
$$

for every $(x, M)$ in $\boldsymbol{X} \times \mathfrak{M}$. Each $h_{m n}(., M)$ is $\mathfrak{R}$-measurable and we always have $h_{m n} \leqq h_{m+1, n}$ and $h_{m n} \leqq h_{m, n+1}$. Note that for every $m, n$, and $M$ we have

$$
\int_{.} h_{m n}(., M) g d \nu=\Sigma_{0 \leqq k \leqq 2^{2 n}} \int_{x \cap E(k, n, M)} k 2^{-i n} H\left(., E_{m}\right) g d \nu
$$

for every $N$ in $\mathfrak{R}$. Because $\left\{x \in X:^{\prime} W(x, M)=\infty\right\}$ is $\nu$-null it is evident that for each $m$ and $M$ we have

$$
\lim _{n} h_{m n}(., M)=H\left(., E_{m}\right)^{\prime} W(., M)
$$

a.e. $\boldsymbol{\nu}$ on $\boldsymbol{X}$. Since $H\left(., E_{m}\right)^{\prime} W(., M) \leqq 2^{m}$ a.e. $\boldsymbol{\nu}$ on $\boldsymbol{X}$ and since $g$ is in $L_{1}^{+}(\nu)$, it follows that $h_{m n}(., M) g$ and $H\left(., E_{m}\right)^{\prime} W(., M) g$ are all in $L_{1}^{+}(\nu)$ and

$$
\lim _{n} \int_{N} h_{m n}(., M) g d \nu=\int_{N} H\left(., E_{m}\right)^{\prime} W(., M) g d \nu
$$

for every $N$ in $\mathfrak{R}, M$ in $\mathfrak{M}$, and $m$ in $Z^{+}$. 
Now, (1), (2), (3), and the monotone convergence theorem imply

$$
\int_{F} g_{m}(., M) d \lambda=\int_{\phi^{-1} F} H\left(., E_{m}\right)^{\prime} W(., M) g d \nu
$$

for every $F$ in $\mathfrak{F}, M$ in $\mathfrak{M}$, and $m$ in $Z^{+}$. We apply the transformation formula for ' $W$ and $f$ to the right member of (4) to obtain

$$
\int_{F} g_{m}(., M) d \lambda=\int_{(\oplus \circ T)^{-1} F \cap M} H\left(., T^{-1} E_{m}\right)(g \circ T) f d \mu
$$

for every $F$ in $\mathfrak{F}, M$ in $\mathfrak{M}$, and $m$ in $Z^{+}$.

We are prepared to prove that ' $U$ is a weighting function for $\Phi \circ T$. In view of .13(a), it is sufficient to prove that each $g_{m}$ is a weighting function for $\Phi \circ T$. Let $m$ be in $Z^{+}$. Let $F$ be any set in $\widetilde{F}$. If we replace $F$ by its complement $C F$ and $M$ by $(\Phi \circ T)^{-1} F$ in (5) we obtain

$$
\int_{C F} g_{m}\left(.,(\Phi \circ T)^{-1} F\right) d \lambda=\int_{\left(\oplus^{\prime} T\right)^{-1} C F \cap\left(\varphi^{\prime} \circ\right)^{-1} F} H\left(., T^{-1} E_{m}\right) f(g \circ T) d \mu=0 ;
$$

therefore $g_{m}\left(.,(\Phi \circ T)^{-1} F\right)=0$ a.e. $\lambda$ on $C F$. Next, suppose that the set $M$ in $\mathfrak{M}$ is the union of a countable number of pairwise disjoint sets $M_{i}$ in $\mathfrak{M}$. Then, for every $F$ in $\mathfrak{F}$, we have, using (4),

$$
\begin{aligned}
\int_{F} g_{m}(., M) d \lambda & =\int_{\Phi^{-1} F} H\left(., E_{m}\right)^{\prime} W(., M) g d \nu \\
& =\int_{\Phi^{-1} F} H\left(., E_{m}\right) \Sigma^{\prime} W\left(., M_{i}\right) g d \nu \\
& =\Sigma \int_{\Phi^{-1} F} H\left(., E_{m}\right)^{\prime} W\left(., M_{i}\right) g d \nu \\
& =\int_{F} \Sigma g_{m}\left(., M_{i}\right) d \lambda .
\end{aligned}
$$

Consequently $g_{m}(., M)=\Sigma g_{m}\left(., M_{i}\right)$ a.e. $\lambda$ on $\boldsymbol{Y}$. Thus $g_{m}$ is a weighting function for $\Phi \circ T$.

Since $f$ is in $J(T), f=0$ a.e. $\mu$ on $T^{-1}\left(X-\cup E_{m}\right)$. Hence (4), (5), and the monotone convergence theorem lead to

$$
\int_{F}{ }^{\prime} U(., M) d \lambda=\int_{\Phi^{-1} F^{\prime}}{ }^{\prime} W(., M) g d \nu=\int_{\left(\odot^{\prime} T\right)-1_{F^{\prime} \cap M}} f(g \circ T) d \mu
$$

for every $F$ in $\mathfrak{F}$ and $M$ in $\mathfrak{M}$. In particular we have

$$
\int_{Y}{ }^{\prime} U(., S) d \lambda=\int_{X}{ }_{X} W(., S) g d \nu=\int_{S} f(g \circ T) d \mu .
$$

We deduce that ' $U(., S)$ is in $L_{1}^{+}(\lambda)$ if and only if ' $W(., S) g$ is in $L_{1}^{+}(\nu)$, or, equivalently, if and only if $f(g \circ T)$ is in $L_{1}^{+}(\mu)$. The final statement of the conclusion of the theorem follows from .11 and (6). 
Definition 16. Assume $\mathrm{A} 0 *-\mathrm{A} 3, \mathrm{~B} 0 *-\mathrm{B} 3$, and $\mathrm{C} 0$ *-C3. Assume ' $W$ is in $J^{\prime}(T),{ }^{\prime} V$ is in $J^{\prime}(\Phi)$, and ' $U$ is in $J^{\prime}(\Phi \circ T)$. ' $U$ is a chain product of ' $W$ and ' $V$ in case there exist functions $f$ in $J(T)$ and $g$ in $J(\Phi)$ such that $f$ is a g.l.b. $f . w g$ is a g.l.b. $f . V$, and $f(g \circ T)$ is a g.l.b. f.U.

Remarks. Assume that ' $W$ is in $J^{\prime}(T),{ }^{\prime} V$ is in $J^{\prime}(\Phi)$, and that ' $U$ is a chain product of ' $W$ and ' $V$. Let $f$ be a g.l.b. $f . W$. and $g$ be a g.l.b. f.V. Suppose that ' $U_{0}$ is in $J^{\prime}(\Phi \circ T)$ and that $h_{0}$ is a g.l.b. $f . U_{0}$. Then ' $U_{0}$ is a chain product of ' $W$ and ' $V$ if and only if ${ }^{\prime} U_{0}(., M)={ }^{\prime} U(., M)$ a.e. $\lambda$ on $Y$ for every $M$ in $\mathfrak{M}$, or, equivalently, if and only if $h_{0}=f(g \circ T)$ a. e. $\mu$ on $S$.

REMARKS 17. Throughout the rest of 1 we consider a "factoring problem," that is, we assume $\mathrm{A} 0 *-\mathrm{A} 3, \mathrm{~B} 0-{ }^{*} \mathrm{~B} 3$, and $\mathrm{C} 0 *-\mathrm{C} 3$ and assume that ' $U$ is in $J^{\prime}(\Phi \circ T)$ and attempt to find conditions under which ' $U$ can be expressed as a chain product of weighting functions ' $W$ in $J^{\prime}(T)$ and ' $V$ in $J^{\prime}(\Phi)$.

Theorem 18. Assume $\mathrm{A} 0-{ }^{*} \mathrm{~A} 3, \mathrm{~B} 0 *-\mathrm{B} 3$, and $\mathrm{C} 0 *-\mathrm{C} 3$. Suppose 'U is in $J^{\prime}(Ф \circ T)$ and let $h$ be a g.l.b. f.U. Then there exist weighting functions ' $W$ in $J^{\prime}(T)$ and ' $V$ in $J^{\prime}(\Phi)$ such that ' $U$ is a chain product of ' $W$ and ' $V$ if and only if there exist functions $f$ in $J(T)$ and $g$ in $J(\Phi)$ such that $h=f(g \circ T)$ a.e. $\mu$ on $\boldsymbol{S}$.

Proof. First assume that ' $U$ is a chain product of ' $W$ in $J^{\prime}(T)$ and ' $V$ in $J^{\prime}(\Phi)$. There exist $f$ in $J(T)$ and $g$ in $J(\Phi)$ such that $f$ is a g.l.b. $f . W, g$ is a g.l.b. $f . V$, and $f(g \circ T)$ is a g.l.b. $f . U$. According to $.6, h=f(g \circ T)$ a. e. $\mu$ on $S$. The converse follows from .8 and .16 .

Remarks 19. Assume $\mathrm{A} 0 *-\mathrm{A} 3, \mathrm{~B} 0 *-\mathrm{B} 3$, and $\mathrm{C} 0$ - $-\mathrm{C} 3$. Assume that ' $U$ is in $J^{\prime}(\Phi \circ T)$. In this setting one may ask whether it is always possible to "factor" ' $U$ as a chain product; that is, one may ask whether it is always possible to obtain ' $W$ in $J^{\prime}(T)$ and ' $V$ in $J(\Phi)$ such that ' $U$ is a chain product of ' $W$ and ' $V$.

The following example reveals that the answer is no.

ExAMPLe 20. Let $R^{n}$ denote real Euclidean $n$-space and let $\lambda_{n}$ denote $n$-dimensional Lebesgue measure. Define sets

$$
\begin{aligned}
& S_{1}=\left\{(u, v) \in R^{2}: u^{2}+(v-4)^{2}<1 \text { and } v \leqq 4\right\}, \\
& S_{2}=\left\{(u, v) \in R^{2}:-1<u<1 \text { and } 4 \leqq v \leqq 5\right\}, \\
& S_{3}=\left\{(u, v) \in R^{2}: u^{2}+(v-5)^{2}<1 \text { and } v \geqq 5\right\}, \quad S=S_{1} \cup S_{2} \cup S_{3}, \\
& \boldsymbol{X}=\left\{(u, v) \in R^{2}: u^{2}+(v-1)^{2}<1\right\}, \text { and } \\
& \boldsymbol{Y}=1-1,1\left[=\left\{u \in R^{2}:-1<u<1\right\} .\right.
\end{aligned}
$$


Let $\mathfrak{M}$ and $\mathfrak{N}$ be the families of all $\lambda_{2}$-measurable subsets of $R^{2}$ which are also subsets of $S$ and $\boldsymbol{X}$ respectively. Let $\Re$ be the family of all $\lambda_{1}$-measurable subsets of $R^{1}$ which are also subsets of $\boldsymbol{Y}$. Let $\mathfrak{U}$ be the family of all Borel sets in $R^{2}$ which are subsets of $X$. Let $\widetilde{F}$ be the family of all Borel sets in $R^{1}$ which are subsets of $\boldsymbol{Y}$. Set $\mu=\lambda_{2}\left|\mathfrak{M}, \nu=\lambda_{2}\right| \Re$, and $\lambda=\lambda_{1} \mid \Re$. Define a function $T$ from $\boldsymbol{S}$ onto $\boldsymbol{X}$ by setting $T(u, v)=(u, v-3)$ if $(u, v)$ is in $S_{1}, T(u, v)=(u, 1)$ if $(u, v)$ is in $S_{2}$, and $T(u, v)=(u, v-4)$ if $(u, v)$ is in $S_{3}$. Define a function $\Phi$ from $\boldsymbol{X}$ onto $\boldsymbol{Y}$ by setting $\Phi(u, v)=u$ for every $(u, v)$ in $X$. $\quad T$ and $\Phi$ are continuous relative to the usual Euclidean topologies. Standard theorems imply that $\mathrm{A} 00^{*}-\mathrm{A} 3, \mathrm{~B} 00^{*} \mathrm{~B} 3$, and $\mathrm{C} 0{ }^{*}-\mathrm{C} 3$ hold with these choices.

Define a function ' $U$ having domain $Y \times \mathfrak{M}$ as follows: If $(y, M)$ is in $\boldsymbol{Y} \times \mathfrak{M}$ then set

$$
{ }^{\prime} U(y, M)=\lambda_{1}\left\{v \in R^{1}:(y, v) \in M\right\} \quad \text { if } \quad\left\{v \in R^{1}:(y, v) \in M\right\}
$$

is $\lambda_{1}$-measurable and put ' $U(y, M)=0$ otherwise. Evidently ' $U$ is a weighting function for $\Phi \circ T$ and $\int_{Y}{ }_{Y} U(., M) d \lambda=\mu M$ for every $M$ in $\mathfrak{M}$; an appeal to the Fubini Theorem is required in the justification of these assertions. Let $F$ be in $\mathfrak{F}$ and $M$ be in $\mathfrak{M}$. If $H$ denotes the characteristic function of $F$ as a subset of $\boldsymbol{Y}$, then

$$
\begin{aligned}
\int_{F^{\prime}}{ }^{\prime} U(., M) d \lambda & =\int_{Y} \lambda_{1}\left\{v \in R^{1}:(y, v) \in M \cap(\Phi \circ T)^{-1} F\right\} d \lambda(y) \\
& =\mu\left[M \cap(\Phi \circ T)^{-1} F\right]=\int_{J I} H \circ(\Phi \circ T) d \mu .
\end{aligned}
$$

Define a real valued function $h$ having domain $S$ by setting $h(s)=1$ for every $s$ in $S$. In view of 11 and (1), ' $U$ is in $J^{\prime}(\Phi \circ T)$ and $h$ is a g.l.b. f.U.

We use .18 to prove that ' $U$ cannot be factored as a chain product. Indeed if it were possible to express ' $U$ as a chain product of ' $W$ in $J^{\prime}(T)$ and ' $V$ in $J^{\prime}(\Phi)$ then by .18 there would be functions $f$ in $J(T)$ and $g$ in $J(\Phi)$ such that $h=f(g \circ T)$ a.e. $\mu$ on $S$.

$$
\text { Set } A=\{(u, v) \in \boldsymbol{X}: v=1\} \text {. }
$$

Since $f$ is in $J(T), f=0$ a.e. $\mu$ on $T^{-1} A=S_{2}$. But then $h=0$ a.e. $\ell^{\ell}$ on $S_{2}$; this is a contradiction.

This example illustrates the following theorem.

Theorem 21. Assume $\mathrm{A} 0 *-\mathrm{A} 3, \mathrm{~B} 0 *-\mathrm{B} 3$, and $\mathrm{C} 0 *-\mathrm{C} 3$. Assume that ' $U$ is in $J^{\prime}(\Phi \circ T)$ and that ' $U$ can be factored as a chain product of weighting functions in $J^{\prime}(T)$ and $J^{\prime}(\bar{T})$. If $A$ is a ע-null set in 2 ir then $U T^{-1} A=0$. 
Proof. Let $h$ be a g.l.b.f.U. By .18, $h$ must be in $J(T)$. The desired conclusion follows at once.

Remarks 22. Assume $\mathrm{A} 0 *-\mathrm{A} 3$. A set $A_{T}$ in $\mathfrak{A}$ is termed an essential $T$-set if it is $\nu$-null and if for every $\nu$-null set $N$ in $\Re$ it is true that $T^{-1}\left(N \cap C A_{T}\right)$ is a $\mu$-null set in $\mathfrak{M}$. It is known [4, Lemma 3, p. 235] that essential $T$-sets always exist. An essential $T$-set is, in effect, a $\nu$-null set whose inverse image under $T$ is maximal.

The theorem in .21 has a partial converse.

Theorem 23. Assume $\mathrm{A} 0 *-\mathrm{A} 3, \mathrm{~B} 0 *-\mathrm{B} 3$, and $\mathrm{C} 0$ *-C3. Assume that ' $U$ is in $J^{\prime}(\Phi \circ T)$ and that $U T^{-1} A=0$ whenever $A$ is any $\nu$-null set in A. Finally assume that there exists an essential $\Phi$-set $F_{\Phi}$ such that $\boldsymbol{\nu}\left(\boldsymbol{X}-\Phi^{-1} F_{\oplus}\right)<\infty$. Then there exist weighting functions ' $W$ in $J^{\prime}(T)$ and ' $V$ in $J^{\prime}(\Phi)$ such that ' $U$ is a chain product of ' $W$ and ' $V$.

Proof. Again we use .18. Let $h$ be a g.l.b.f.U. Set $f=h$ and let $g$ be the characteristic function of $\boldsymbol{X}-\Phi^{-1} F_{\Phi}$ as a subset of $\boldsymbol{X}$. Hence $f$ is in $L_{1}^{+}(\mu)$; since $U T^{-1} A=0$ whenever $A$ is a $\nu$-null set in $\mathfrak{A}$, it follows that $f=h$ is in $J(T)$. An elementary argument involving the properties of essential $\Phi$-sets shows that $g$ is in $J(\Phi)$ and that $h=f(g \circ T)$ a.e. $\mu$ on $S$. An appeal to .18 completes the proof.

Corollary 24. Assume A0*-A3, B0*-B3, and $\mathrm{C} 0 *-\mathrm{C} 3$. Assume that ' $U$ is in $J^{\prime}(\Phi \circ T)$ and that $U T^{-1} A=0$ whenever $A$ is any $\nu$-null set in $\mathfrak{A}$. Finally assume that $\nu \boldsymbol{X}<\infty$. Then there exist weighting functions ' $W$ in $J^{\prime}(T)$ and ' $V$ in $J^{\prime}(\Phi)$ such that ' $U$ is a chain product of ' $W$ and ' $V$.

In .25 we present an example which shows that the condition that $\nu\left(X-\Phi^{-1} F_{\phi}\right)$ be finite cannot be dropped entirely from .23 and which suggests the difficulty involved when $\nu\left(X-\Phi^{-1} F_{\emptyset}\right)$ is infinite.

ExAmple 25. Let $\boldsymbol{S}, \boldsymbol{X}$, and $\boldsymbol{Y}$ each be the open interval $] 1,+\infty[$ and let $\mathfrak{M}, \mathfrak{R}$, and $\Re$ each be the family of all $\lambda_{1}$-measurable subsets of $R^{1}$ which are also subsets of $\boldsymbol{S}=\boldsymbol{X}=\boldsymbol{Y}$. Let $\mathfrak{A}$ and $\mathfrak{F}$ each be the family of all Borel sets in $R^{1}$ which are subsets of $S$. Set $\mu=\nu=\lambda=\lambda_{1} \mid \mathfrak{M}$. Define a function $T$ from $\boldsymbol{S}$ onto $\boldsymbol{X}$ by $T(s)=s$ for each $s$ in $S$; set $\Phi=T$. It is clear that the hypotheses $\mathrm{A} 0^{*}$-A3, $\mathrm{B} 0 *-\mathrm{B} 3, \mathrm{C} 0 *-\mathrm{C} 3$ hold for the above choices of $S, X$, etc. Now define a real valued function $h$ having domain $S$ by putting $h(s)=s^{-2}$ for every $s$ in $S$. Obviously $h$ is in $J(\Phi \circ T)$ and so, in view of .8 , there exists ' $U$ in $J^{\prime}(\Phi \circ T)$ such that $h$ is a g.l.b. f.U. Also, $T^{-1} A$ is $\mu$-null whenever $A$ is any $\nu$-null set in $\mathfrak{A}$. Hence the hypotheses of .23 are 
satisfied except for the assumption that $\nu\left(X-\Phi^{-1} F_{\Phi}\right)$ be finite. Now $h$ cannot be written as a product $f g$ of functions $f$ in $L_{1}^{+}(\mu)$ and $g$ in $L_{1}^{+}(\mu)$ (cf. 13,28 b) of [8]). Hence, in view of .18, ' $U$ cannot be factored as a chain product of weighting functions ' $W$ in $J^{\prime}(T)$ and $' V$ in $J^{\prime}(\Phi)$.

2. Chain products in other settings. Formulas for the transformation of integrals in a measure-theoretic context have been established in [10] and [4]. These results are developed in settings which are special instances of the setting established by imposing the hypotheses A0-A3. We can prove theorems in these other settings which are analogous to those proved in $\S 1$. These results are, in a sense, special cases of those in $\S 1$; for they are derivable from the results of $\S 1$ together with key theorems which relate the weighting functions of $\S 1$ to the weight (and quasi-weight) functions of [10] and the weighing functions of [4].

Now we recast the results of $\S 1$ in the setting first described in [10].

REMARKS 1. The transformation theory presented in [10] is developed in a setting which is established by imposing certain hypotheses. Among these are statements denoted by $\mathrm{H} 1-\mathrm{H} 9, \mathrm{H} 9$ *, $\mathrm{H} 11-\mathrm{H} 13$, and $\mathrm{H}^{*}$. See $\S 1$ and $\S 7$ of [10], $\S 1$ of [3], $\S 5$ of [4], and $\S 1$ of [5] for complete statements of these hypotheses. Actually, one can follow what ensues simply by reading H1-H9 in $\S 1$ of [10], H11H12 in $\S 7$ of [10], and $\S 9$ of [10]. Assume H1-H8. Then, according to $\S 5$ of [4], the statements A0-A3 hold if we put $\boldsymbol{X}=\boldsymbol{S}^{\prime}, \mathfrak{N}=\mathfrak{M}^{\prime}$, $\nu=\mu^{\prime}, \mathfrak{A}=\mathfrak{B}^{\prime}$, and $\mathbb{S}=\mathfrak{D}$. Whenever H1-H8 are in effect we shall always make these notational identifications.

Definitions 2. Assume H1-H8. $T$ is $A C W$ if there is a function $f$ in $L_{1}^{+}(\mu)$ such that $W D=\int_{S^{\prime}} W^{\prime}\left(s^{\prime}, D\right) d \mu^{\prime}\left(s^{\prime}\right)=\int_{D} f d \mu$ for every $D$ in D); such a function $f$ is termed a greatest lower bound function for the weights on $\mathfrak{D}$ induced by $W^{\prime}$-briefly, a g.l.b. $f . W$. (If $T$ is $A C W$ and $f$ is a g.l.b. $f . W$. then, according to 4.10 of [10], a "transformation formula" holds for $W^{\prime}$ and $f$.) Define $J^{\prime \prime}\left(T, \mu, \mu^{\prime}, \mathfrak{D}\right)=J^{\prime \prime}(T)$ to be the set of all weight functions $W^{\prime}$ for $T$ for which $T$ is $A C W$ (see $\S 1$ and 3.4 of $[10])$. Define $J^{\prime *}\left(T, \mu, \mu^{\prime}, \mathscr{D}\right)=J^{\prime *}(T)$ to be the set of all quasi-weight functions $W^{\prime}$ for $T$ for which $T$ is $A C W$ (see 5.1 of [4]). Connections between these sets and $J^{\prime}(T)$ appear in .3.

REMARKS 3. For the reader's convenience we list several results from other papers. We assume that H1-H8 are satisfied. 
(i) $J^{\prime \prime}(T) \subset J^{\prime *}(T)$.

(ii) Assume also $\mathrm{H}^{*}$. If $W^{\prime}$ is in $J^{\prime *}(T)$ then there exists ${ }_{0} W^{\prime}$ in $J^{\prime \prime}(T)$ such that ${ }_{0} W^{\prime}\left(s^{\prime}, D\right)=W^{\prime}\left(s^{\prime}, D\right)$ a.e. $\mu^{\prime}$ on $\boldsymbol{S}^{\prime}$ for every $D$ in $\supseteqq$.

(iii) Assume H1-H8. If ' $W$ is in $J^{\prime}(T)$ and if $f$ is a g.l.b. $f . W$. then ${ }^{\prime} W \mid S^{\prime} \times \mathscr{D}$ is in $J^{\prime *}(T)$ and $f$ is a g.l.b. $f . W . \| \mathfrak{D}$. (We use $W \mid \mathfrak{D}$ here because a quasi-weight function attaches weights only to (D), not to all of $\mathfrak{M}$.) If $W^{\prime}$ is in $J^{\prime *}(T)$ and if $f$ is a g.1.b. $f . W$. then there exists ${ }^{\prime} W$ in $J^{\prime}(T)$ such that $W^{\prime}={ }^{\prime} W \mid \boldsymbol{S}^{\prime} \times \mathfrak{M}$ and $f$ is a g.l.b. $f . W$.

It was observed in 5.1 of [4] that (i) holds. (ii) is a consequence of 1.3 of [5]. For (iii) we appeal to 5.5 and 5.6 of [4].

Remarks 4. Assume H1-H8. As in $\S 1$, we shall be concerned with the composition $T^{\prime \prime}=T^{\prime} \circ T$ of two transformations. As before, we shall need two additional lists of statements. Let $\mathrm{H}^{\prime} \mathrm{T}^{\prime}-\mathrm{H} \mathrm{T}^{\prime}$, $\mathrm{H} 9 * \mathrm{~T}^{\prime}, \mathrm{H} 11 \mathrm{~T}^{\prime}-\mathrm{H} 13 \mathrm{~T}^{\prime}$, and $\mathrm{H}^{*} \mathrm{~T}^{\prime}$ be the statements obtained respectively from $\mathrm{H} 1-\mathrm{H} 9, \mathrm{H} 9 *$, $\mathrm{H} 11-\mathrm{H} 13$, and $\mathrm{H}^{*}$ by making these replacements: Replace $\{\boldsymbol{S}, \mathfrak{M}, \boldsymbol{\mu}\}$ by $\left\{\boldsymbol{S}^{\prime}, \mathfrak{M}^{\prime}, \mu^{\prime}\right\},\left\{\boldsymbol{S}^{\prime}, \mathfrak{M}^{\prime}, \mu^{\prime}\right\}$ by $\left\{\boldsymbol{S}^{\prime \prime}, \mathfrak{M}^{\prime \prime}, \mu^{\prime \prime}\right\}, T$ by $T^{\prime}$, (D) by $\mathfrak{D}^{\prime}, \mathfrak{B}^{\prime}$ by $\mathfrak{B}^{\prime \prime}, \mathfrak{D}^{\prime}$ by $\mathfrak{D}^{\prime \prime}\left(T^{\prime}\right), W^{\prime}$ by $V^{\prime}$, $\mathfrak{S}$ by $\mathfrak{S}^{\prime}$, by $v$, and $\mathfrak{D}_{c}$ by $\mathfrak{D}_{c}^{\prime}$. Full statements of $\mathrm{H}^{\prime} \mathrm{T}^{\prime}-\mathrm{H}^{\prime} \mathrm{T}^{\prime}$ and $\mathrm{H}^{\prime} 11 \mathrm{~T}^{\prime}-\mathrm{H} 13 \mathrm{~T}^{\prime}$ appear in Chapter IV in [6], although there are some minor differences in notation. Also let $\mathrm{H}_{1} \mathrm{~T}^{\prime \prime}-\mathrm{H} 9 \mathrm{~T}^{\prime \prime}, \mathrm{H} 9^{*} \mathrm{~T}^{\prime \prime}, \mathrm{H} 11 \mathrm{~T}^{\prime \prime}-\mathrm{H} 13 \mathrm{~T}^{\prime \prime}$, and $\mathrm{H}^{*} \mathrm{~T}^{\prime \prime}$ be the statements obtained respectively from $\mathrm{H} 1-\mathrm{H} 9, \mathrm{H} 9 *$, H11-H13, and $\mathrm{H}^{*}$ by making these replacements: Replace $\left\{\boldsymbol{S}^{\prime}, \mathfrak{M} \mathfrak{N}^{\prime}, \boldsymbol{\mu}^{\prime}\right\}$ by $\left\{\boldsymbol{S}^{\prime \prime}, \mathfrak{M}^{\prime \prime}, \boldsymbol{\mu}^{\prime \prime}\right\}$, $T$ by $T^{\prime \prime}=T^{\prime} \circ T, \mathfrak{B}^{\prime}$ by $\mathfrak{B}^{\prime \prime}, \mathfrak{S}^{\prime}$ by $\mathfrak{S}^{\prime \prime}\left(T^{\prime \prime}\right), W^{\prime}$ by $U^{\prime}$, and $w$ by $u$. Full statements of $\mathrm{H}_{1} \mathrm{~T}^{\prime \prime}-\mathrm{H} \mathrm{T}^{\prime \prime}$ and $\mathrm{H} 11 \mathrm{~T}^{\prime \prime}-\mathrm{H} 13 \mathrm{~T}^{\prime \prime}$ appear in Chapter $V$ in [6].

Now assume that all of the statements $\mathrm{H} 1-\mathrm{H} 8$ and $\mathrm{H} 1 \mathrm{~T}^{\prime}-\mathrm{H} 8 \mathrm{~T}^{\prime}$ hold. Then all of the statements $\mathrm{H}_{1} \mathrm{~T}^{\prime \prime}-\mathrm{H} 8 \mathrm{~T}^{\prime \prime}$ are satisfied if one assumes merely that $\mathrm{H}_{5} \mathrm{~T}^{\prime \prime}, \mathrm{H} 7 \mathrm{~T}^{\prime \prime}$, and H8T" hold, that $\left(T^{\prime \prime}\right)^{-1} \mathrm{~S}^{\prime \prime} \subset \mathfrak{M}$, and that $T^{\prime \prime} \mathfrak{D} \subset \mathfrak{M}^{\prime \prime}$ (cf. Chapter $V$ in [6]). Note also that if H11

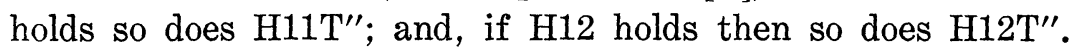

In 2.5-2-12 we shall assume that $\mathrm{H} 1-\mathrm{H} 8, \mathrm{H} 1 \mathrm{~T}^{\prime}-\mathrm{H}_{8} \mathrm{~T}^{\prime}$, and $\mathrm{H} 1 \mathrm{~T}^{\prime \prime}-\mathrm{H} 8 \mathrm{~T}^{\prime \prime}$ are in effect. The statements A0-A3, B0-B3, and CO-C3 all hold if we put $\boldsymbol{X}=\boldsymbol{S}^{\prime}, \mathfrak{N}=\mathfrak{M}^{\prime}, \nu=\mu^{\prime}, \Phi=T^{\prime}, \mathfrak{U}=\mathfrak{B}^{\prime}, \boldsymbol{Y}=\boldsymbol{S}^{\prime \prime}, \mathfrak{R}=\mathfrak{M}^{\prime \prime}, \lambda=$ $\mu^{\prime \prime}, \mathfrak{F}=\mathfrak{B}^{\prime \prime}, \mathfrak{S}=\mathfrak{D}$, and $\mathfrak{S}=\mathfrak{D}^{\prime}$. Throughout 2.5-2.14 we shall always make these notational identifications.

Definition 5. Assume $W^{\prime}$ is in $J^{* *}(T), V^{\prime}$ is in $J^{* *}\left(T^{\prime}\right)$, and $U^{\prime}$ is in $J^{\prime *}\left(T^{\prime \prime}\right)$. $U^{\prime}$ is a chain product of $W^{\prime}$ and $V^{\prime}$ if there exist functions $f$ in $J(T)$ and $g$ in $J\left(T^{\prime}\right)$ such that $f$ is a g.l.b. $f . W, g$ is a g.l.b. $f . V$, and $f(g \circ T)$ is a g.l.b. $f . U$.

Lemma 6. Assume $W^{\prime}$ is in $J^{*^{\prime}}(T) V^{\prime}$ is in $J^{*^{\prime}}\left(T^{\prime}\right), U^{\prime}$ is in 
$J^{* \prime}\left(T^{\prime \prime}\right)$, and that $U^{\prime}$ is a chain product of $W^{\prime}$ and $V^{\prime}$. Let $f$ be a g.l.b. $f . W, g$ be a g.l.b. $f . V$, and $h$ be a g.l.b.f.U. Then there exist 'W in $J^{\prime}(T),{ }^{\prime} V$ in $J^{\prime}\left(T^{\prime}\right)$, and ' $U$ in $J^{\prime}\left(T^{\prime \prime}\right)$ such that

$$
W^{\prime}={ }^{\prime} W\left|\boldsymbol{S}^{\prime} \times \mathfrak{D}, V^{\prime}={ }^{\prime} V\right| \boldsymbol{S}^{\prime \prime} \times \mathfrak{D}^{\prime}, U^{\prime}={ }^{\prime} U \mid \boldsymbol{S}^{\prime \prime} \times \mathfrak{D},
$$

$f$ is a g.l.b. $f . W, g$ is $a$ g.l.b. $V, h$ is $a$ g.l.b. $f . V$ and ' $U$ is a chain product of ' $W$ and ' $V$.

Now assume that ' $W$ is in $J^{\prime}(T),{ }^{\prime} V$ is in $J^{\prime}\left(T^{\prime}\right),{ }^{\prime} U$ is in $J^{\prime}\left(T^{\prime \prime}\right)$, and that ' $U$ is a chain product of ${ }^{\prime} W$ and ${ }^{\prime} V$. Then $U^{\prime}={ }^{\prime} U \mid \boldsymbol{S}^{\prime \prime} \times \mathfrak{D}$ is a chain product of $W^{\prime}={ }^{\prime} W \mid \boldsymbol{S}^{\prime} \times \mathfrak{D}$ and $V^{\prime}={ }^{\prime} V \mid \boldsymbol{S}^{\prime \prime} \times \mathfrak{D}^{\prime}$. If $f$ is a g.l.b. f. $W, g$ is a g.l.b. $f . V$, and $h$ is a g.l.b. $f . U$, then $f$ is a g.l.b. $f . W \mid \mathfrak{D}, g$ is a g.l.b. $f . V \mid \mathfrak{D}^{\prime}$, and $h$ is a g.l.b. $f . U \mid \mathfrak{D}$.

Proof. This lemma follows from 1.16, .5 and .3(iii).

THEorem 7. (i) Assume that $W^{\prime}$ is in $J^{\prime *}(T)$ and that $V^{\prime}$ is in $J^{*}\left(T^{\prime}\right)$. Let $f$ be a g.l.b. $f . W$. and $g$ be a g.l.b. $f . V$. Then there exists $U^{\prime}$ in $J^{\prime *}\left(T^{\prime \prime}\right)$ such that $f(g \circ T)$ is a g.l.b. f.U. if and only if $g W^{\prime}(., S)$ is in $L_{1}^{+}\left(\mu^{\prime}\right)$, or, equivalently, if and only if $f(g \circ T)$ is in $L_{1}^{+}(\mu)$.

(ii) Assume also $H^{*} T^{\prime \prime}$. Then (i) is true if $J^{\prime *}$ is replaced throughout by $\mathrm{J}^{\prime \prime}$.

Proof. (i) follows from 1.15, .3(iii), and .6. .3(i), .3(ii), and (i) imply (ii).

REMARKS 8. Just as before, we consider the "factoring problem;" that is, we assume that $U^{\prime}$ is in $J^{\prime *}\left(T^{\prime \prime}\right)$ (respectively, $J^{\prime \prime}\left(T^{\prime \prime}\right)$ ) and we attempt to determine whether $U^{\prime}$ can be expressed as a chain product of quasi-weight functions (respectively, weight functions) for for $T$ and $T^{\prime}$.

THEOREM 9. (i) Assume that $U^{\prime}$ is in $J^{\prime *}\left(T^{\prime \prime}\right)$ and let $h$ be a g.l.b.f.U. Then there exist $W^{\prime}$ in $J^{\prime *}(T)$ and $V^{\prime}$ in $J^{* *}\left(T^{\prime}\right)$ such that $U^{\prime}$ is a chain product of $W^{\prime}$ and $V^{\prime}$ if and only if there exist functions $f$ in $J(T)$ and $g$ in $J\left(T^{\prime}\right)$ such that $h=f(g \circ T)$ a.e. $\mu$ on $\boldsymbol{S}$.

(ii) Assume also $H^{*}$ and $H^{*} T^{\prime}$. Then (i) is true if $J^{\prime *}$ is replaced throughout by $J^{\prime \prime}$.

Proof. For (i) we appeal to 1.18, .3(iii), and .6. The proof of (ii) depends on .3(i), (i), and .3(ii).

THeorem 10. (i) Assume that $U^{\prime}$ is in $J^{\prime *}\left(T^{\prime \prime}\right)$ and that $U^{\prime}$ can be factored as a chain product of functions $W^{\prime}$ in $J^{\prime *}(T)$ and $V^{\prime}$ in $J^{*}\left(T^{\prime}\right)$. Let $h$ be a g.l.b. f.U. If $B^{\prime}$ is a $\mu^{\prime}$-null set in $\mathfrak{B}^{\prime}$ it is true 
that $h=0$ a.e. $\mu$ on $T^{-1} B^{\prime}$; i.e., $h$ is in $J(T)$.

(ii) Statement (i) remains true if $J^{\prime *}$ is replaced throughout by $J^{\prime \prime}$.

Proof. To prove (i), we appeal to 1.21 and .6. (ii) follows from (i) and .3(i).

As before, the above theorem admits a partial converse.

Theorem 11. (i) Assume that $U^{\prime}$ is in $J^{\prime *}\left(T^{\prime \prime}\right)$ and let $h$ be a g.l.b.f.U. Assume that $h$ is in $J(T)$ and suppose that

$$
\mu^{\prime}\left(\boldsymbol{S}^{\prime}-T^{\prime-1} E^{\prime \prime}\right)<\infty
$$

where $E^{\prime \prime}$ is some essential $T^{\prime}$-set. Then there exist functions $W^{\prime}$ in $J^{\prime *}(T)$ and $V^{\prime}$ in $J^{\prime *}\left(T^{\prime}\right)$ such that $U^{\prime}$ is a chain product of $W^{\prime}$ and $V^{\prime}$.

(ii) Assume also $H^{*}$ and $H^{*} T^{\prime}$. Then (i) remains true if $J^{\prime *}$ is replaced throughout by $\mathrm{J}^{\prime \prime}$.

Proof. 1.23, .3(iii), and .6. imply (i). Again, (ii) is a consequence of .3(i), .3(ii), and (i).

CoRollary 12. (i) Assume that $U^{\prime}$ is in $J^{* *}\left(T^{\prime \prime}\right)$ and let $h$ be a g.l.b.f.U. Assume that $h$ is in $J(T)$ and suppose that $\mu^{\prime} \mathbf{S}^{\prime}<\infty$. Then there exist functions $W^{\prime}$ in $J^{*}(T)$ and $V^{\prime}$ in $J^{*}\left(T^{\prime}\right)$ such that $U^{\prime}$ is a chain product of $W^{\prime}$ and $V^{\prime}$.

(ii) Assume also $H^{*}$ and $H^{*} T^{\prime}$. Then (i) remains true if $J^{*}$ is replaced throughout by $J^{\prime \prime}$.

EXAMPLE 13. We present a modified version of the example of 1.20. Maintaining the notation of 1.20 we put $\boldsymbol{S}^{\prime}=\boldsymbol{X}, \mathfrak{M}^{\prime}=\mathfrak{N}$, $\mu^{\prime}=\nu, \mathfrak{B}^{\prime}=\mathfrak{U}, \boldsymbol{S}^{\prime \prime}=\boldsymbol{Y}, \mathfrak{M}^{\prime \prime}=\Re, \mu^{\prime \prime}=\lambda, T^{\prime}=\Phi, \mathfrak{B}^{\prime \prime}=\mathfrak{F}$, and $T^{\prime \prime}=$ $T^{\prime} \circ T$. Let $\mathfrak{D}$ and $\mathfrak{D}^{\prime}$ consist of the connected open subsets of $S$ and $\boldsymbol{S}^{\prime}$ respectively. Let $\mathfrak{S}^{\prime}, \mathfrak{S}^{\prime \prime}\left(T^{\prime}\right)$, and $\mathfrak{D}^{\prime \prime}\left(T^{\prime \prime}\right)$ consist respectively of those subsets of $\boldsymbol{S}^{\prime}, \boldsymbol{S}^{\prime \prime}$, and $\boldsymbol{S}^{\prime \prime}$ which are respectively open relative to $R^{2}, R^{1}$ and $R^{1}$. With these choices it follows from 9.1-9.8 of [10] that all of the standard hypothese $\mathrm{H} 1-\mathrm{H} 8, \mathrm{H} 1 \mathrm{~T}^{\prime}-\mathrm{H} 8 \mathrm{~T}^{\prime}$, and $\mathrm{H} 1 \mathrm{~T}^{\prime \prime}-\mathrm{H}_{8} \mathrm{~T}^{\prime \prime}$ are satisfied. Let $\mathfrak{D}_{c}$ and $\mathfrak{D}_{c}^{\prime}$ be respectively the families of all bounded sets in $\mathfrak{D}$ and $\mathfrak{D}^{\prime}$ which are the interiors of polygons having rational vertices. Then $\mathrm{H}^{*}, \mathrm{H}^{*} \mathrm{~T}^{\prime}$, and $\mathrm{H}^{*} \mathrm{~T}^{\prime \prime}$ are satisfied. Now set

$$
U^{\prime}={ }^{\prime} U \mid S^{\prime \prime} \times \mathfrak{D},
$$

where ' $U$ is defined as in 1.20. As in 6.1 of [2], $U^{\prime}$ is in $J^{\prime \prime}\left(T^{\prime \prime}\right)$. In view of $1.7, .6$, and $1.20, U^{\prime}$ cannot be factored as a chain product of functions in $J^{\prime *}(T)$ and $J^{\prime *}\left(T^{\prime}\right)$. 
EXAMPLE 14. We shall consider the example of 1.25 in the present setting. Retaining the notation of 1.25 we put $\boldsymbol{S}^{\prime}=\boldsymbol{X}, \mathfrak{M}^{\prime}=\mathfrak{R}$, $\mu^{\prime}=\nu, \mathfrak{B}^{\prime}=\mathfrak{A}, \boldsymbol{S}^{\prime \prime}=\boldsymbol{Y}, \mathfrak{M}^{\prime \prime}=\Re, \mu^{\prime \prime}=\lambda, T^{\prime}=\Phi, \mathfrak{B}^{\prime \prime}=\mathfrak{F}$, and $T^{\prime \prime}=$ $T^{\prime} \circ T$. Let $\mathfrak{D}$ and $\mathfrak{D}^{\prime}$ each consist of all open subintervals of $] 1,+\infty[$. Let $\mathfrak{D}^{\prime}, \mathfrak{S}^{\prime \prime}\left(T^{\prime}\right)$, and $\mathfrak{S}^{\prime}\left(T^{\prime \prime}\right)$ each consist of all open subsets of $] 1,+\infty[$. With these choices it follows again from $9.1-9.8$ of [10] that all of the standard hypotheses $\mathrm{H} 1-\mathrm{H} 8, \mathrm{H}_{1} \mathrm{~T}^{\prime}-\mathrm{H} 8 \mathrm{~T}^{\prime}$, and $\mathrm{H} 1 \mathrm{~T}^{\prime \prime}-\mathrm{H} 8 \mathrm{~T}^{\prime \prime}$ are satisfied. Let $\mathfrak{D}_{c}$ and $\mathfrak{D}_{c}^{\prime}$ each consist of all bounded subintervals of ] $1,+\infty$ [ having rational endpoints. Then $\mathrm{H}^{*}, \mathrm{H}^{*} \mathrm{~T}^{\prime}$, and $\mathrm{H}^{*} \mathrm{~T}^{\prime \prime}$ are satisfied. Define $h$ as in 1.25. By 2.5 of [5] there exists $U^{\prime}$ in $J^{\prime \prime}\left(T^{\prime \prime}\right)$ such that $h$ is a g.l.b. f.U. The hypotheses of .11 are fulfilled except for the requirement that $\mu^{\prime}\left(S^{\prime \prime}-T^{\prime-1} E^{\prime \prime}\right)$ be finite. However, just as in $1.25, U^{\prime}$ cannot be factored as a chain product of $W^{\prime}$ in $J^{\prime *}(T)$ and $V^{\prime}$ in $J^{\prime *}\left(T^{\prime}\right)$.

Now we can also recast the results of $\S 1$ in the setting of [4]. We can obtain a list of theorems and examples which are analogous in virtually every way to those just presented in .1-.14; of course, the concept of "(quasi-) weight function" is replaced throughout by that of "weighing function." This list is omitted because its contents are obvious to anyone who is familiar with [4] and quite meaningless to anyone who is not familiar with [4].

3. Chain products for a special type of weight functions. Now we discuss the main application of the theorem in 1.15.

Reichelderfer introduced and discussed a special class of weight functions in $\S 7$ of [10]. This special class includes all of the (nonnegative) admissible multiplicity functions - the forerunners of weight functions-which play a key role in [9]. In this section we ask whether chain products of weight functions of this special class are also of this special type. Then we describe briefly the relationship between the resulting theorem and earlier theorems dealing with similar situations.

Remarks 1. Assume H1-H8 and H11-H12. Within this context we can define the special class $\Omega(T)$ of weight functions for $T$ which was referred to above. First, as in 7.1 of [10], we define $\subseteq$ to be the family of all subsets $S$ of $S$ for each of which there is a point $s$ in $S$ such that $S=\cap\{D \in \mathfrak{D}: s \in D\}$. According to 7.2 of [10], $\mathfrak{S}$ partitions $S$. And, $\mathrm{H} 12$ asserts that for each $S$ in $S$ the set $T S=\{T(s): s \in S\}$ contains but one element. Now define $\&$ to be the family of all subsets $E$ of $S$ each of which is the union of sets in $\subseteq$. It was observed in 7.6 of [10] that $\mathbb{F}$ is a $\sigma$-field which includes $\mathfrak{D}$. If $W^{\prime}$ is a weight function for $T$ and if $w$ is a non negative, extended real valued function having domain $\mathfrak{\subseteq}$, then we say that $W^{\prime}$ is gen- 
erated by $w$ or that $w$ generates $W^{\prime}$ if

$$
W^{\prime}\left(s^{\prime}, D\right)=\Sigma\left\{w(S): S \in \mathbb{S}, T S=s^{\prime}, S \subset D\right\}
$$

for all $\left(s^{\prime}, D\right)$ in $S^{\prime} \times \mathfrak{D}, \Omega(T)$ is defined to be the set of all weight functions $W^{\prime}$ for $T$ for each of which there exists a non negative, extended real valued function $w$ having domain $\subseteq$ such that $W^{\prime}$ is generated by $w$. The functions of $Q(T)$ are discussed at length in $\S 7$ of [10].

Remarks 2. Assume H1-H8 and H11-H12. Define

$$
\Omega^{\prime}(T)=\Omega(T) \cap J^{\prime \prime}(T) .
$$

Thus $\Omega^{\prime}(T)$ is the set of all weight functions $W^{\prime}$ in $Q(T)$ for which $T$ is $A C W$.

Now suppose that $W^{\prime}$ is in $\Omega^{\prime}(T)$ and assume that $W^{\prime}$ is generated by $w$. Define a function $W^{*}$ having domain $S^{\prime} \times \mathfrak{M} \cap \mathbb{E}$ as follows: For every $\left(s^{\prime}, E\right)$ in $S^{\prime} \times \mathfrak{M} \cap \mathbb{E}$ put

$$
W^{*}\left(s^{\prime}, E\right)=\Sigma\left\{w(S): S \in \subseteq, T S=s^{\prime}, S \subset E\right\} .
$$

Thus $W^{*}$ is an extension of $W^{\prime}$.

It was noted in 7.8 of [10] that $W^{*}$ has these properties:

(i) If $E$ is in $M \cap F$ and if $s^{\prime}$ is in $S^{\prime}-T E$ then $W^{*}\left(s^{\prime}, E\right)=0$.

(ii) For each $s^{\prime}$ in $\boldsymbol{S}^{\prime}$ the function $W^{*}\left(s^{\prime}\right.$, .) is a measure on $\mathfrak{M} \cap \mathfrak{F}$.

Frazier has proved [6, Chapter III] that $W^{*}$ has these additional properties:

(iii) For each $E$ in $\mathfrak{M} \cap \mathbb{F}$ the function $W^{*}(., E)$ is in $L_{1}^{+}(\mu)$.

(iv) If $f$ is a g.l.b. $f . W$. then $\int_{S^{\prime}} W^{*}(., E) d \mu^{\prime}=\int_{E} f d \mu$ for every $E$ in $\mathfrak{M} \cap \mathfrak{F}$.

(v) Let $f$ be a g.l.b. $f . W$. Then, the transformation formula holds for $W^{*}$ and $f$. More precisely, the following is true.

Let $E$ be in $\mathfrak{M} \cap \mathfrak{F}$. Suppose that $H^{\prime}$ is a real valued $\mathfrak{M}^{\prime}$-measurable function having domain $\boldsymbol{S}^{\prime}$. Then the function $\left(H^{\prime} \circ T\right) f$ is $\mathfrak{M}$-measurable and $H^{\prime} W^{*}(., E)$ is $\mathfrak{M}^{\prime}$-measurable. Suppose also that $\left(H^{\prime} \circ T\right) f$ is $\mu$-integrable over $E$ or that $H^{\prime} W^{*}(., E)$ is in $L_{1}\left(\mu^{\prime}\right)$. Then $\left(H^{\prime} \circ T\right) f$ is $\mu$-integrable over $E, H^{\prime} W^{*}(., E)$ is in $L_{1}\left(\mu^{\prime}\right)$, and

$$
\int_{S^{\prime}} H^{\prime} W^{*}(., E) d \mu^{\prime}=\int_{E}\left(H^{\prime} \circ T\right) f d \mu .
$$

Note. The function $W^{*}$ defined above is termed a*-extension of $W^{\prime}$ Since $W^{\prime}$ may have several generators, it may conceivably have several *-extensions. However, one can prove, using (v), that two 
*-extensions agree except on $\mu^{\prime}$-null sets.

Theorem 3. Assume H1-H8 and H11-H12. Assume that $W^{\prime} i s$ in $\Omega^{\prime}(T)$ and let $f$ be a g.l.b. $f . W$. Let $w$ be any generator of $W^{\prime}$ and let $W^{*}$ be the ${ }^{*}$-extension of $W^{\prime}$ determined by $w$ as in .2 . Then therc exists ' $W$ in $J^{\prime}(T)$ such that $W^{*}={ }^{\prime} W \mid \boldsymbol{S}^{\prime} \times \mathfrak{M} \cap$ (5; moreover $f$ is a g.l.b. $f$. $W$.

Proof. By 2.6 of [10], $f$ is in $J(T)$. By 1.8 there exists ' $W_{0}$ in $J^{\prime}(T)$ such that $f$ is a g.l.b. $f . W_{0}$. If $E$ is in $\mathfrak{M} \cap \mathbb{F}$ and if $H^{\prime}$ is the characteristic function of a set $M^{\prime}$ in $\mathfrak{M}^{\prime}$ then $.2(\mathrm{v})$ implies

$$
\int_{M \prime}{ }^{\prime} W_{0}(., E) d \mu^{\prime}=\int_{E}\left(H^{\prime} \circ T\right) f d \mu=\int_{M \prime} W^{*}(., E) d \mu^{\prime} .
$$

Hence ${ }^{\prime} W_{0}(., E)=W^{*}(., E)$ a.e. $\mu^{\prime}$ on $S^{\prime}$ for every $E$ in $\mathfrak{M} \cap \mathfrak{\xi}$. We now obtain the desired ' $W$ from ' $W$ o by altering the latter on certain $\mu^{\prime}$-null sets.

4. In order to be able to discuss chain products of functions $W^{\prime}$ in $\Omega^{\prime}(T)$ and $V^{\prime}$ in $\Omega^{\prime}\left(T^{\prime}\right)$, we shall assume that all of the standard hypotheses H1-H8, H11-H12, H1 $\mathrm{T}^{\prime}-\mathrm{H} 8 \mathrm{~T}^{\prime}, \mathrm{H} 11 \mathrm{~T}^{\prime}-\mathrm{H} 12 \mathrm{~T}^{\prime}, \mathrm{H} 1 \mathrm{~T}^{\prime \prime}-\mathrm{H} 8 \mathrm{~T}^{\prime \prime}$, and $\mathrm{H} 11 \mathrm{~T}^{\prime \prime}-\mathrm{H} 12 \mathrm{~T}^{\prime \prime}$ are in effect.

To discuss H11T' and H12T' we must define the set $\mathfrak{S}^{\prime} . \mathfrak{S}^{\prime}$ is, of course, analogous to $\widetilde{S}$; that is, $\widetilde{S}^{\prime}$ is the family of all subsets $S^{\prime}$ of $S^{\prime}$ for each of which there is a point $s^{\prime}$ in $S^{\prime}$ such that

$$
S^{\prime}=\cap\left\{D^{\prime} \in \mathscr{D}^{\prime}: s^{\prime} \in D^{\prime}\right\} \text {. }
$$

Now define $\mathfrak{F}^{\prime}$ in terms of $\mathfrak{S}^{\prime}$ just as $\mathfrak{F}$ was defined in terms of $\mathfrak{S}$.

Theorem 5. Assume H1-H8, H11-H12, H1T'-H8T', H11T'-H12T', $\mathrm{H} 1 \mathrm{~T}^{\prime \prime}-\mathrm{H} 8 \mathrm{~T}^{\prime \prime}$, and H11T"-H12T". Assume that $W^{\prime}$ is in $\Omega^{\prime}(T)$ and that $V^{\prime}$ is in $\Omega^{\prime}\left(T^{\prime}\right)$. Let $f$ be a g.l.b. $f . W$. and $g$ be a g.l.b. $f . V$.

Now let $w$ be an nonnegative, extended real valued function having domain $\subseteq$ which generates $W^{\prime}$ and let $v$ be a nonnegative, extended real valued function having domain $\widetilde{S}^{\prime}$ which generates $V^{\prime}$. Let $c$ be any function having domain $\widetilde{S}^{\prime}$ with the property that $c\left(S^{\prime}\right)$ is a point in $S^{\prime}$ for each $S^{\prime}$ in $\mathfrak{S}^{\prime}$. (Thus $c$ is a choice function.) Define a nonnegative, extended real valued function $p$ having domain $\boldsymbol{S}^{\prime}$ as follows: If $s^{\prime}$ is in $c\left(\mathcal{S}^{\prime}\right)$ define $p\left(s^{\prime}\right)$ to be $v\left(S^{\prime}\right)$ where $S^{\prime}$ is the unique member of $\widetilde{S}^{\prime}$ such that $c\left(S^{\prime}\right)=s^{\prime}$; otherwise, if $s^{\prime}$ is not in $c\left(\mathfrak{S}^{\prime}\right)$, put $p\left(s^{\prime}\right)=0$. Next, define a nonnegative, extended real valued function $u$ having domain $\subseteq$ by setting $u(S)=w(S) p(T S)$ for every $S$ in $\subseteq$ (where $\infty .0=0)$.

Then $u$ generates a function $U^{\prime}$ in $\Omega\left(T^{\prime \prime}\right) . \quad U^{\prime}(., S)$ is in $L_{1}^{+}\left(\mu^{\prime \prime}\right)$ 
if and only if $g W^{\prime}(., S)$ is in $L_{1}^{+}\left(\mu^{\prime}\right)$, or, equivalently, if and only if $f(g \circ T)$ is in $L_{1}^{+}(\mu)$. If $U^{\prime}(., S)$ is in $L_{1}^{+}\left(\mu^{\prime \prime}\right)$ then $U^{\prime}$ is in $\Omega^{\prime}\left(T^{\prime \prime}\right)$ and $U^{\prime}$ is a chain product of $W^{\prime}$ and $V^{\prime}$.

Proof. As in .2 we introduce the *-extension $W^{*}$ of $W^{\prime}$ determined by $w$ and the *-extension $V^{*}$ of $V^{\prime}$ determined by $v$. Define a non negative, extended real valued function $U^{*}$ having domain $\boldsymbol{S}^{\prime \prime} \times \mathfrak{M} \cap \mathbb{F}$ by setting

$$
U^{*}\left(s^{\prime \prime}, E\right)=\Sigma\left\{u(S): S \in \mathfrak{S}, S \subset E, T^{\prime \prime} S=s^{\prime \prime}\right\}
$$

for every $\left(s^{\prime \prime}, E\right)$ in $S^{\prime \prime} \times \mathfrak{M} \cap \mathfrak{F}$. A routine argument involving the definitions of the terms at hand proves

$$
U^{*}\left(s^{\prime \prime}, E\right)=\Sigma\left\{p\left(s^{\prime}\right) W^{*}\left(s^{\prime}, E\right): s^{\prime} \in \boldsymbol{S}^{\prime}, T^{\prime} s^{\prime}=s^{\prime \prime}\right\}
$$

for every $\left(s^{\prime \prime}, E\right)$ in $S^{\prime \prime} \times \mathfrak{M} \cap \mathbb{S}$; we omit the details. According to .3 there is a weighting function ' $W_{0}$ in $J^{\prime}(T)$ such that

$$
W^{*}={ }^{\prime} W_{0} \mid \boldsymbol{S}^{\prime} \times \mathfrak{M} \cap \mathbb{E}
$$

and such that $f$ is a g.l.b. $f . W_{0}$. Similarly there is a weighting function ' $V$ in $J^{\prime}\left(T^{\prime}\right)$ such that $V^{*}={ }^{\prime} V \mid S^{\prime \prime} \times \mathfrak{M}^{\prime} \cap \mathcal{F}^{\prime}$ and such that $g$ is a g.l.b. $f . V$. By 7.6 of [10], $\mathfrak{D}^{\prime} \subset \mathcal{F}^{\prime}$; hence $\mathfrak{M}^{\prime} \cap \mathfrak{S}^{\prime}$ is a $\sigma$-field containing $\mathfrak{D}^{\prime}$. A simple argument using $\mathrm{H}_{4} \mathrm{~T}^{\prime}$ and 7.6 of [10] (cf. 2.8 of [3]) shows that there is a function ' $W$ in $J^{\prime}(T)$ such that

(i ) ${ }^{\prime} W(., M)={ }^{\prime} W_{\circ}(., M)$ a.e. $\mu^{\prime}$ on $S^{\prime}$ for every $M$ in $\mathfrak{M}$;

(ii) $f$ is a g.l.b. $f . W$;

(iii) for each $M$ in $\mathfrak{M}$ the function ' $W(., M)$ is $\mathfrak{M}^{\prime} \cap \widetilde{F}^{\prime}$-measurable. For each pair of positive integers $k$ and $n$ and for every $M$ in $M$ define subsets $E^{\prime}(k, n, M)$ and $B^{\prime}(k, n, M)$ of $S^{\prime}$ by putting

$$
E^{\prime}(k, n, M)=\left\{s^{\prime} \in \boldsymbol{S}^{\prime}: k 2^{-n} \leqq W_{0}\left(s^{\prime}, M\right)<(k+1) 2^{-n}\right\}
$$

and

$$
B^{\prime}(k, n, M)=\left\{s^{\prime} \in \boldsymbol{S}^{\prime}: k 2^{-n} \leqq{ }^{\prime} W\left(s^{\prime}, M\right)<(k+1) 2^{-n}\right\} .
$$

Each set $E^{\prime}(k, n, M)$ is in $\mathfrak{M}^{\prime}$ and, in view of (iii) above, each set $B^{\prime}(k, n, M)$ is in $\mathfrak{M}^{\prime} \cap \mathbb{F}^{\prime}$ !

Now we introduce the particular chain product ' $U$ of ' $W$ and ' $V$ which is given by 1.15 . Thus for each set $M$ in $M$ there is a $\mu^{\prime \prime}$-null set $K^{\prime \prime}(M)$ in $\mathfrak{M}^{\prime \prime}$ such that the sequence

$$
\left\{\Sigma_{k} k 2^{-n \prime} V\left(s^{\prime \prime}, B^{\prime}(k, n, M)\right)\right\}_{n}
$$

is nondecreasing and such that

$$
{ }^{\prime} U\left(s^{\prime \prime}, M\right)=\lim _{n} \Sigma_{k} k 2^{-n \prime} V\left(s^{\prime \prime}, B^{\prime}(k, n, M)\right)
$$


for all $\mathrm{s}^{\prime \prime}$ in $\boldsymbol{S}^{\prime \prime}-K^{\prime \prime}(M)$; moreover ${ }^{\prime} U\left(\mathrm{~s}^{\prime \prime}, M\right)=0$ if $s^{\prime \prime}$ is in $K^{\prime \prime}(M)$. The set $B^{\prime}(k, n, M)$ is in $\mathfrak{M}^{\prime} \cap \mathfrak{F}^{\prime}$ for every $k, n$ and $M$ and so ${ }^{\prime} V\left(s^{\prime \prime}, B^{\prime}(k, n, M)\right)=V^{*}\left(s^{\prime \prime}, B^{\prime}(k, n, M)\right)$ for every $s^{\prime \prime}$ in $S^{\prime \prime}, k, n$, and $M$. Now define

$$
S_{+}^{\prime}=\cup\left\{S^{\prime} \in \mathfrak{S}^{\prime}: v\left(S^{\prime}\right)>0\right\}
$$

and

$$
S_{\infty}^{\prime}=\left\{s^{\prime} \in \boldsymbol{S}^{\prime}: W^{\prime}\left(s^{\prime}, \boldsymbol{S}\right)=+\infty\right\} .
$$

Since $W^{\prime}(., S)$ is in $L_{1}^{+}\left(\mu^{\prime}\right)$ it is clear that $S_{\infty}^{\prime}$ is $\mu^{\prime}$-null. At this point, we assert

$$
{ }^{\prime} U\left(s^{\prime \prime}, E\right)=U^{*}\left(s^{\prime \prime}, E\right) \text { a.e. } \mu^{\prime \prime} \text { on } S^{\prime \prime}, \quad E \in \mathfrak{M} \cap \leftleftarrows \text {. }
$$

For every $k, n$, and $E$ in $\mathfrak{M} \cap \mathbb{E}$ the symmetric difference

$$
E^{\prime}(k, n, E) \Delta B^{\prime}(k, n, E)
$$

is $\mu^{\prime}$-null. For each $E$ in $\mathfrak{M} \cap \mathbb{F}$ define

$$
X^{\prime \prime}(E)=T^{\prime}\left\{S_{\longleftarrow}^{\prime} \cap\left[S_{\infty}^{\prime} \cup \cup_{k, n}\left(E^{\prime}(k, n, E) \Delta B^{\prime}(k, n, E)\right)\right]\right\} ;
$$

according to 7.24 of [10], the set $X^{\prime \prime}(E)$ is $\mu^{\prime \prime}$-null. We shall prove (3) by showing that ${ }^{\prime} U\left(s^{\prime \prime}, E\right)=U^{*}\left(s^{\prime \prime}, E\right)$ whenever $E$ is in $\mathfrak{M} \cap \mathbb{E}$ and $s^{\prime \prime}$ is in $\boldsymbol{S}^{\prime \prime}-\left(K^{\prime \prime}(E) \cup X^{\prime \prime}(E)\right)$.

Thus let $E$ be a fixed set in $\mathfrak{M} \cap \mathbb{F}$ and let $s^{\prime \prime}$ be a fixed point in $S^{\prime \prime}-\left(K^{\prime \prime}(E) \cup X^{\prime \prime}(E)\right)$. First we establish

$$
U^{*}\left(s^{\prime \prime}, E\right) \leqq ' U\left(s^{\prime \prime}, E\right) \text {. }
$$

We may as well assume $U^{*}\left(s^{\prime \prime}, E\right)>0$. Let $t$ be a positive number such that $t<U^{*}\left(s^{\prime \prime}, E\right)$. In view of (1) there exist distinct points ${ }_{1} s^{\prime}, \cdots,{ }_{m} s^{\prime}$ in $S^{\prime}$ such that $T^{\prime}{ }_{i} s^{\prime}=s^{\prime \prime}$ for $i=1, \cdots, m$ and such that $t<\Sigma_{1 \leqq i \leqq m} p\left({ }_{i} s^{\prime}\right) W^{*}\left({ }_{i} s^{\prime}, E\right)$. We may assume that each of the extended real numbers $p\left({ }_{i} s^{\prime}\right) W^{*}\left({ }_{i} s^{\prime}, E\right)$ is positive. Several cases must be considered. First if $W^{*}\left({ }_{i} s^{\prime}, E\right)=+\infty$ for some $i$ then ${ }_{i} s^{\prime}$ is in $S_{+}^{\prime} \cap S_{\infty}^{\prime}$ and so $s^{\prime \prime}$ is in $X^{\prime \prime}(E)$; this is contradictory to our assumption. Thus we may assume that all of the numbers $W^{*}\left({ }_{i} s^{\prime}, E\right)$ are finite and positive. There is a positive integer $N$ such that $2^{-N^{*}}<W^{*}\left({ }_{i} s^{\prime}, E\right)$ for $i=1, \cdots, m$. Hence for every positive integer $n \geqq N$ there are positive integers $k_{n 1}, \cdots, k_{n m}$ such that ${ }_{i} s^{\prime}$ is in $E^{\prime}\left(k_{n i}, n, E\right)$ for $i=1, \cdots, m$. Now either $p\left({ }_{i} s^{\prime}\right)=+\infty$ for some $i$ or else the numbers $p\left({ }_{i} s^{\prime}\right), i=1, \cdots, m$, are all finite and positive. First assume $p\left({ }_{i} s^{\prime}\right)=$ $+\infty$ for some $i$. Since $s^{\prime \prime}$ is not in $X^{\prime \prime}(E)$ it is clear that ${ }_{i} s^{\prime}$ is in $B^{\prime}\left(k_{N i}, N, E\right)$. If ${ }_{i} S^{\prime}$ is the unique member of $\mathfrak{S}^{\prime}$ which contains ${ }_{i} s^{\prime}$ then ${ }_{i} S^{\prime} \subset B^{\prime}\left(k_{N i}, N, E\right)$ (because $B^{\prime}\left(k_{N i}, N, E\right)$ is in $\left(\xi^{\prime}\right)$. Therefore $+\infty=k_{N i} 2^{-N} p\left({ }_{i} s^{\prime}\right) \leqq k_{N i} 2^{-N} V^{*}\left(s^{\prime \prime}, B^{\prime}\left(k_{N i}, N, E\right)\right) \leqq ' U\left(s^{\prime \prime}, E\right)$, inasmuch 
as $s^{\prime \prime}$ is not in $K^{\prime \prime}(E)$. Thus $t<+\infty={ }^{\prime} U\left(s^{\prime \prime}, E\right)$ in this case. Finally assume that each of the numbers $p\left({ }_{i} s^{\prime}\right)$ is finite. Pick a positive integer $n$ sufficiently large that $n \geqq N$ and

$$
t+2^{-n} \Sigma_{1 \leqq i \leqq m} p\left({ }_{i} s^{\prime}\right)<\Sigma_{1 \leqq i \leqq m} p\left({ }_{i} s^{\prime}\right) W^{*}\left({ }_{i} s^{\prime}, E\right) .
$$

We have

$$
\Sigma_{1 \leqq i \leqq m} p\left({ }_{i} s^{\prime}\right) W^{*}\left({ }_{i} s^{\prime}, E\right) \leqq \Sigma_{1 \leqq i \leqq m} p\left({ }_{i} s^{\prime}\right) 2^{-n} k_{n i}+2^{-n} \Sigma_{1 \leqq i \leqq m} p\left({ }_{i} s^{\prime}\right) .
$$

Let ${ }_{1} S^{\prime}, \cdots,{ }_{m} S^{\prime}$ be respectively the sets in $S^{\prime}$ which contain the points ${ }_{1} s^{\prime}, \cdots,{ }_{m} s^{\prime}$. Since each $p\left({ }_{i} s^{\prime}\right)$ is positive, the sets ${ }_{1} S^{\prime}, \cdots,{ }_{m} S^{\prime}$ must be distinct. As before, it follows that ${ }_{i} S^{\prime} \subset B^{\prime}\left(k_{n i}, n, E\right)$ for $i=1, \cdots, m$. Consequently, in view of (2),

$$
\begin{aligned}
& \Sigma_{1 \leqq i \leqq m} p\left({ }_{i} s^{\prime}\right) 2^{-n} k_{n i}=\Sigma_{1 \leqq i \leqq m} k_{n i} 2^{-n} v\left({ }_{i} S^{\prime}\right) \\
& \leqq \Sigma_{k} k 2^{-n} V^{*}\left(s^{\prime \prime}, B^{\prime}(k, n, E)\right) \leqq ' U\left(s^{\prime \prime}, E\right) \text {. }
\end{aligned}
$$

It follows from (5) that $t<^{\prime} U\left(s^{\prime \prime}, E\right)$ in this case also. Thus we have proved that $t<^{\prime} U\left(s^{\prime \prime}, E\right)$ in each of the two cases which can arise. Hence (4) follows. Next we shall prove

$$
' U\left(s^{\prime \prime}, E\right) \leqq U^{*}\left(s^{\prime \prime}, E\right)
$$

where the point $\left(s^{\prime \prime}, E\right)$ is still fixed as above. To prove (6), we may assume ' $U\left(s^{\prime \prime}, E\right)>0$. Let $t$ be a positive number for which $t<^{\prime} U\left(s^{\prime \prime}, E\right)$. By (2) there is a positive integer $n$ such that

$$
t<\Sigma_{k} k 2^{-n \prime} V\left(s^{\prime \prime}, B^{\prime}(k, n, E)\right) \text {. }
$$

Thus there are positive integers $k_{1}, \cdots, k_{p}$ such that

$$
t<\Sigma_{1 \leqq q \leqq p} k_{q} 2^{-n \prime} V\left(s^{\prime \prime}, B^{\prime}\left(k_{q}, n, E\right)\right)
$$

and

$$
k_{q} 2^{-n \prime} V\left(s^{\prime \prime}, B^{\prime}\left(k_{q}, n, E\right)\right)>0
$$

for $q=1, \cdots, p$. Recall that ' $V\left(s^{\prime \prime}, B^{\prime}\left(k_{q}, n, E\right)\right)=V^{*}\left(s^{\prime \prime}, B^{\prime}\left(k_{q}, n, E\right)\right)$ for $q=1, \cdots, p$. Thus for $q=1, \cdots, p$ there exist distinct sets ${ }_{q 1} S^{\prime}, \cdots,{ }_{q n} S^{\prime}$ in $\subseteq^{\prime}$ such that ${ }_{q j} S^{\prime} \subset B^{\prime}\left(k_{q}, n, E\right), v\left({ }_{q j} S^{\prime}\right)>0$, and $T^{\prime}{ }_{q j} S^{\prime}=s^{\prime \prime}$ for each $q$ and $j$ and such that

$$
t<\Sigma_{1 \leqq q \leqq p} k_{q} 2^{-n}\left\{\Sigma_{1 \leqq j \leqq n q} v\left(_{q j} S^{\prime}\right)\right\} .
$$

For $q=1, \cdots, p$ and $j=1, \cdots, n_{q}$ put ${ }_{q j} s^{\prime}=c\left({ }_{q j} S^{\prime}\right)$. Since $s^{\prime \prime}$ is not in $X^{\prime \prime}(E)$ it follows that ${ }_{q j} s^{\prime}$ is in $E^{\prime}\left(k_{q}, n, E\right)$ for $q=1, \cdots, p$ and $j=1, \cdots, n_{q}$. Hence $k_{q} 2^{-n} \leqq{ }^{\prime} W_{0}\left({ }_{q j} s^{\prime}, E\right)=W^{*}\left({ }_{q j} s^{\prime}, E\right)$ for every $q$ and $j$. As a result we must have

$$
t<\Sigma_{1 \leqq q \leqq p} \Sigma_{1 \leqq j \leqq n_{q}} p\left({ }_{q j} s^{\prime}\right) W^{*}\left({ }_{q j} s^{\prime}, E\right) .
$$


The sets ${ }_{q j} S^{\prime}, q=1, \cdots, p$ and $j=1, \cdots, n_{q}$, are distinct; hence the points ${ }_{q j} s^{\prime}, q=1, \cdots, p$ and $j=1, \cdots, n_{q}$, are distinct. It follows from (1) that $t<U^{*}\left(s^{\prime \prime}, E\right)$. The proof of (6) is complete. With (4) and (6) established, it is clear that (3) holds.

First of all, it follows from (3) and from $\mathrm{H}_{2} \mathrm{~T}^{\prime \prime}$ that the function $U^{*}(., E)$ must be $\mathfrak{M}^{\prime \prime}$-measurable for every $E$ in $\mathfrak{M} \cap \mathfrak{F}$. Define $U^{\prime}=U^{*} \mid S^{\prime \prime} \times \mathfrak{D}$. Then, according to 7.9 of $[10], U^{\prime}$ is in $\Omega\left(T^{\prime \prime}\right)$; of course $U^{\prime}$ is generated by $u$. The remaining conclusions of the theorem follow from (3) and 1.15.

REMARKs 6. Let $T$ and $T^{\prime}$ be functions (transformations) from suitable regions in $R^{n}$ into $R^{n}$ which are absolutely continuous in the Banach sense. In V. 4 of [11] Reichelderfer has given necessary and sufficient conditions in order that the function $T^{\prime} \circ T$ be absolutely continuous in the Banach sense. These conditions are direct generalizations of those given in the one-and two-dimensional situations mentioned in the introduction, there is a corresponding "chain rule" as well. Frazier has proved in Chapter X of [6] that Theorem V.4 of [11] is a consequence of the following assertion (see IX.8 of [6]):

Assume that the hypotheses of 3.5 above all hold. Now suppose in addition that for each $D$ in $\mathfrak{D}$ the set $\left\{W^{\prime}\left(s^{\prime}, D\right): s^{\prime} \in \boldsymbol{S}^{\prime}\right\}$ is countable and that $\widetilde{S}^{\prime}$ consists precisely of the "singleton" subsets $\left\{s^{\prime}\right\}$ of $\boldsymbol{S}^{\prime}$, i.e., $\mathfrak{S}^{\prime}=\left\{\left\{s^{\prime}\right\}: s^{\prime} \in \boldsymbol{S}^{\prime}\right\}$. Define a nonnegative, extended real valued function $u$ having domain $\subseteq$ by setting $u(S)=w(S) v(\{T S\})$ for every $S$ in $\subseteq$. Then $u$ generates a function $U^{\prime}$ in $Q\left(T^{\prime \prime}\right)$. $U^{\prime}(., S)$ is in $L_{1}^{+}\left(\mu^{\prime \prime}\right)$ if and only if $g W^{\prime}(., S)$ is in $L_{1}^{+}\left(\mu^{\prime}\right)$, or, equivalently, if and only if $f(g \circ T)$ is in $L_{1}^{+}(\mu)$. If $U^{\prime}(., S)$ is in $L_{1}^{+}\left(\mu^{\prime \prime}\right)$ then $U^{\prime}$ is in $Q^{\prime}\left(T^{\prime \prime}\right)$ and $U^{\prime}$ is a chain product of $W^{\prime}$ and $V^{\prime}$.

Obviously this assertion is a special case of 3.5 (even though the proofs are markedly different).

Frazier has given other specific formulas for chain products $U^{\prime}$ of weight functions $W^{\prime}$ in $J^{\prime \prime}(T)$ and $V^{\prime}$ in $J^{\prime \prime}\left(T^{\prime}\right)$. These formulas apply when the weight functions involved are of certain special types; they appear in VI.7, VII.7, and VIII.9 of [6]. Given $W^{\prime}$ in $J^{\prime \prime}(T)$ and $V^{\prime}$ in $J^{\prime \prime}\left(T^{\prime}\right)$ of any of these appropriate types, one can extend them to weighting functions ' $W$ and ' $V$ as in 2.3(iii) and then define $' U$ as in 1.15. After some computation it becomes evident that the formula for ' $U\left(s^{\prime \prime}, D\right)$ given in 1.15 reduces effectively in each case to Frazier's formula in the sense that the formulas differ only for certain sets of measure zero.

The principal results in [11] are Theorems IV.5.1 and IV.5.2. One might expect that these results can be viewed as special instances 
of 3.5 (or possibly of 1.15 or 2.7). Such is not the case however because the admissible multiplicity functions and essential jacobians of IV.5.1 and IV.5.2-these are the functions which correspond to $W^{\prime}$ and $f$ respectively - are extended real valued but not necessarily nonnegative. Let us suppose that the main results of [10], [4], and the present paper would remain true if the functions $W^{\prime}, V^{\prime},{ }^{\prime} W$, $f$, etc. were allowed to assume negative values as well as nonnegative values; it is likely that IV.5.1 and IV.5.2 of [11] would then be special cases of such an extended version of 3.5. J. Brooks and P. Reichelderfer have recently proved Jordan decomposition theorems for "signed weight functions." These theorems should make it possible to extend many of the principal results of [10], [4], and the present paper to the cases in which the functions $W^{\prime}, V^{\prime}, f$, etc. are allowed to assume negative values.

\section{REFERENCES}

1. N. Bary, Mémoire sur la représentation finite des fonctions continues, Math. Annalen 103 (1930), 185-248 and 598-653.

2. R. W. Chaney, Decomposition theorems for weight functions in the transformation theory for measure space, Rendiconti del Circolo Matematico di Palermo 15 (1966), 98-128.

3. - Measurability theorems in the transformation theory for measure space, Rendiconti del Circolo Matematico di Palermo 14 (1965), 309-323.

4. - On the transformation of integrals in measure space, Pacific J. Math. 19 (1966), 229-242.

5. - Some theorems concerning the class of weight functions in the transformation theory for measure space (submitted for publication).

Palermo).

6. T. Frazier, On the product of absolutely continuous transformations of measure spaces, Ph. D. Thesis, The Ohio State University.

7. R. G. Helsel and N. Levine, Absolutely continuous product transformations of the plane, Duke Math. J. 19 (1952), 595-604.

8. E. Hewitt and K. Stromberg, Real and Abstract Analysis, Springer-Verlag, New York, (1965).

9. T. Rado and P. Reichelderfer, Continuous Transformations in Analysis, SpringerVerlag, Berlin, Gottingen, Heidelberg, 1955.

10. P. Reichelderfer, A transformation theory for measure space, Rendiconti del Circolo Matematico di Palermo 10 (1961), 283-313.

11. On the product of absolutely continuous transformations in Euclidean n-space, Rendiconti del Circolo Matematico di Palermo 5 (1956), 5-42.

Received March 25, 1966, and in revised form April 21, 1967.

Western Washington State College

AND

University of California, Santa Barbara 



\section{PACIFIC JOURNAL OF MATHEMATICS}

\section{EDITORS}

H. ROYDEN

Stanford University

Stanford, California

\section{J. P. Jans}

University of Washington

Seattle, Washington 98105

\section{J. DUGUNDJI}

Department of Mathematics University of Southern California Los Angeles, California 90007

\section{RICHARD ARENS}

University of California

Los Angeles, California 90024

\section{ASSOCIATE EDITORS}

E. F. BECKENBACH

B. H. NEUManN

F. WOLF

K. YosIDA

\section{SUPPORTING INSTITUTIONS}

UNIVERSITY OF BRITISH COLUMBIA CALIFORNIA INSTITUTE OF TECHNOLOGY UNIVERSITY OF CALIFORNIA MONTANA STATE UNIVERSITY UNIVERSITY OF NEVADA NEW MEXICO STATE UNIVERSITY OREGON STATE UNIVERSITY UNIVERSITY OF OREGON OSAKA UNIVERSITY UNIVERSITY OF SOUTHERN CALIFORNIA
STANFORD UNIVERSITY

UNIVERSITY OF TOKYO UNIVERSITY OF UTAH WASHINGTON STATE UNIVERSITY UNIVERSITY OF WASHINGTON AMERICAN MATHEMATICAL SOCIETY CHEVRON RESEARCH CORPORATION TRW SYSTEMS NAVAL WEAPONS CENTER 


\section{Pacific Journal of Mathematics}

\section{Vol. 25, No. $1 \quad$ September, 1968}

Glen Eugene Bredon, Cosheaves and homology................... 1

Robin Ward Chaney, A chain rule for the transformation of integrals in

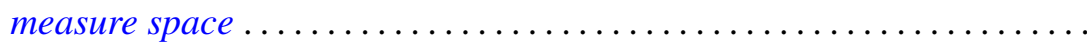

Colin W. Clark, On relatively bounded perturbations of ordinary differential operators................................... 59

John Edwin Diem, A radical for lattice-ordered rings.............. 71

Zeev Ditzian, On a class of convolution transforms ................ 83

Dennis Garoutte and Paul Adrian Nickel, A note on extremal properties characterizing weakly $\lambda$-valent principal functions............. 109

Shwu-Yeng Tzeng Lin, Fixed point properties and inverse limit spaces . . . 117

John S. Lowndes, Some dual series equations involving Laguerre polynomials ................................. 123

Kirti K. Oberai, Sum and product of commuting spectral operators ....... 129

J. N. Pandey and Armen H. Zemanian, Complex inversion for the generalized convolution transformation..................... 147

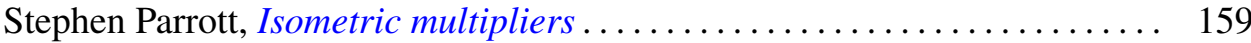

Manoranjan Prasad, Note on an extreme form .................. 167

Maciej Skwarczyński, A representation of a bounded function as infinite product in a domain with Bergman-Shilov boundary surface ......... 177

John C. Taylor, The Šilov boundary for a lattice-ordered semigroup ...... 185 Donald Reginald Traylor and James Newton Younglove, On normality and pointwise paracompactness ............................. 193

L. Tzafriri, Quasi-similarity for spectral operators on Banach spaces ... 